
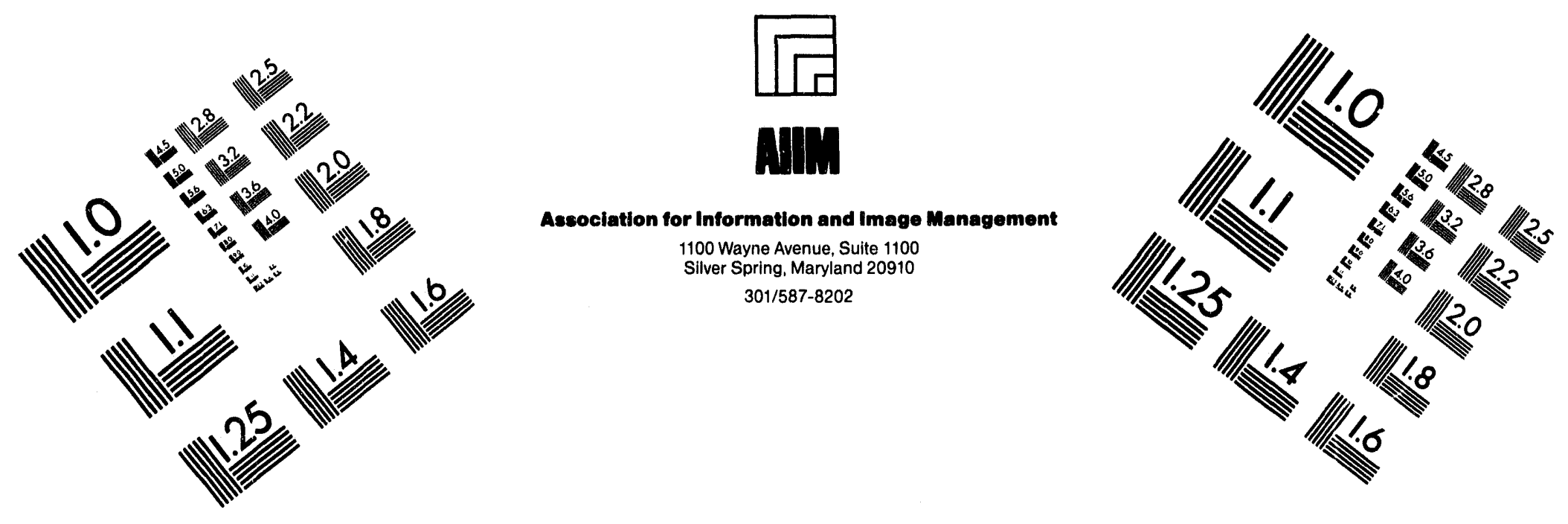

\title{
Centimeter
}

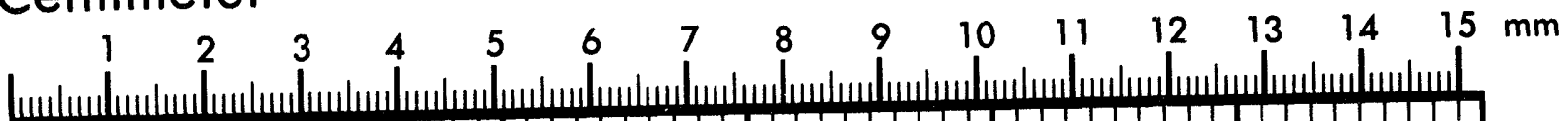

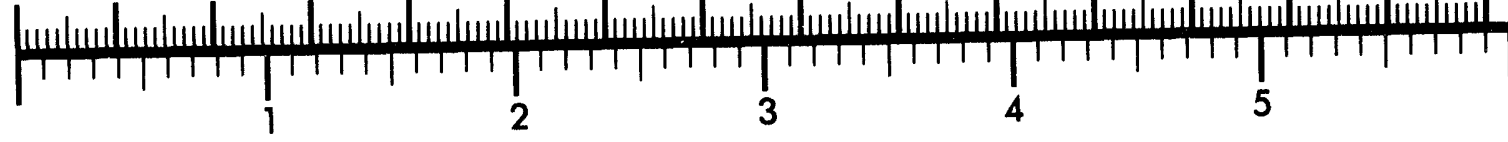
Inches
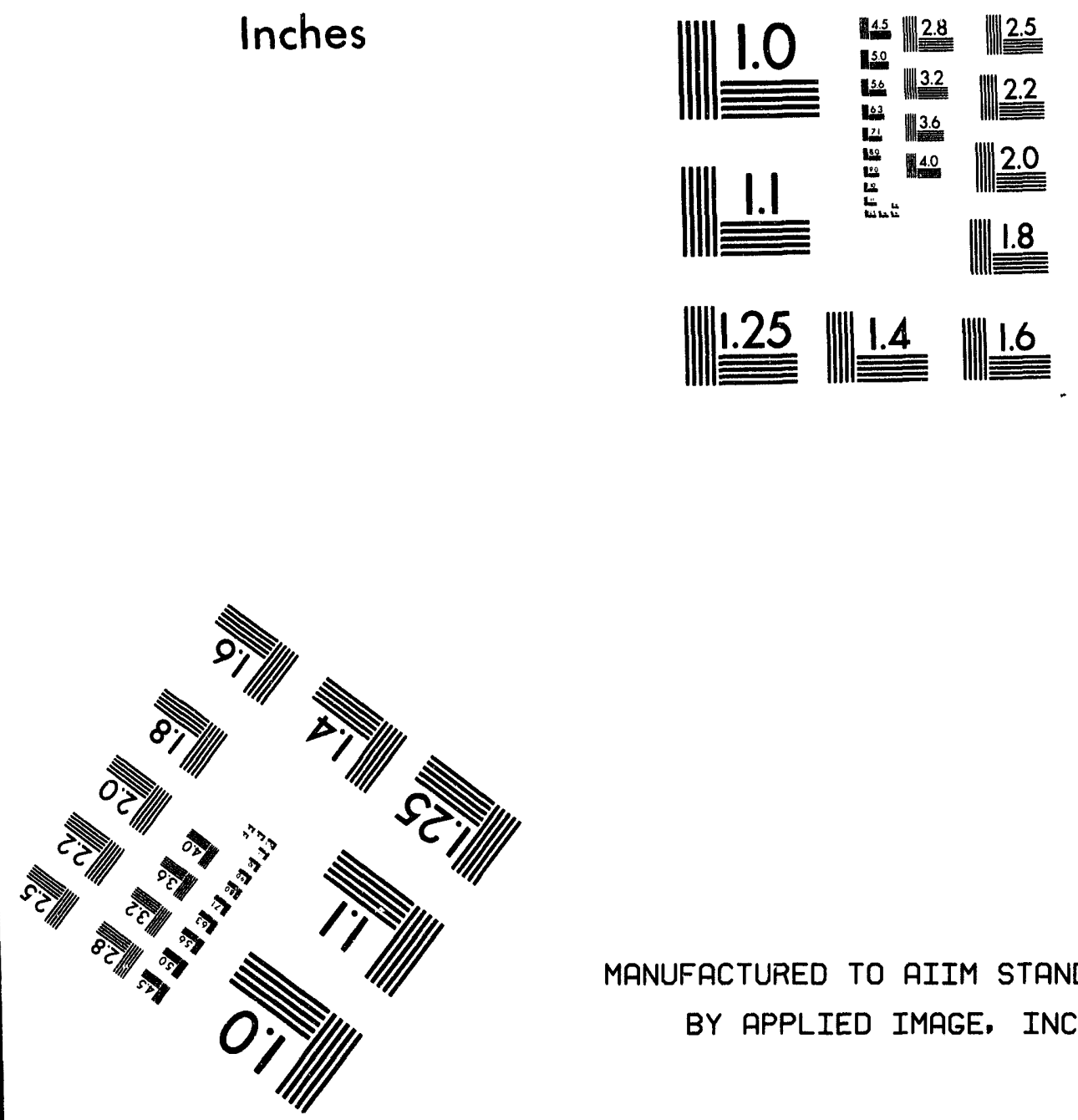

MANUFACTURED TO AIIM STANDARDS BY APPLIED IMAGE, INC.

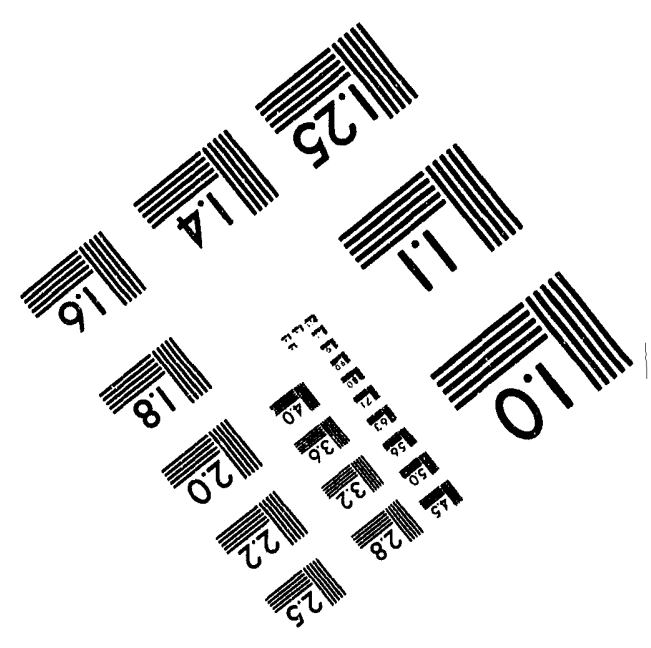




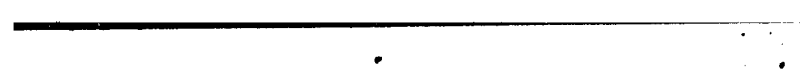

:
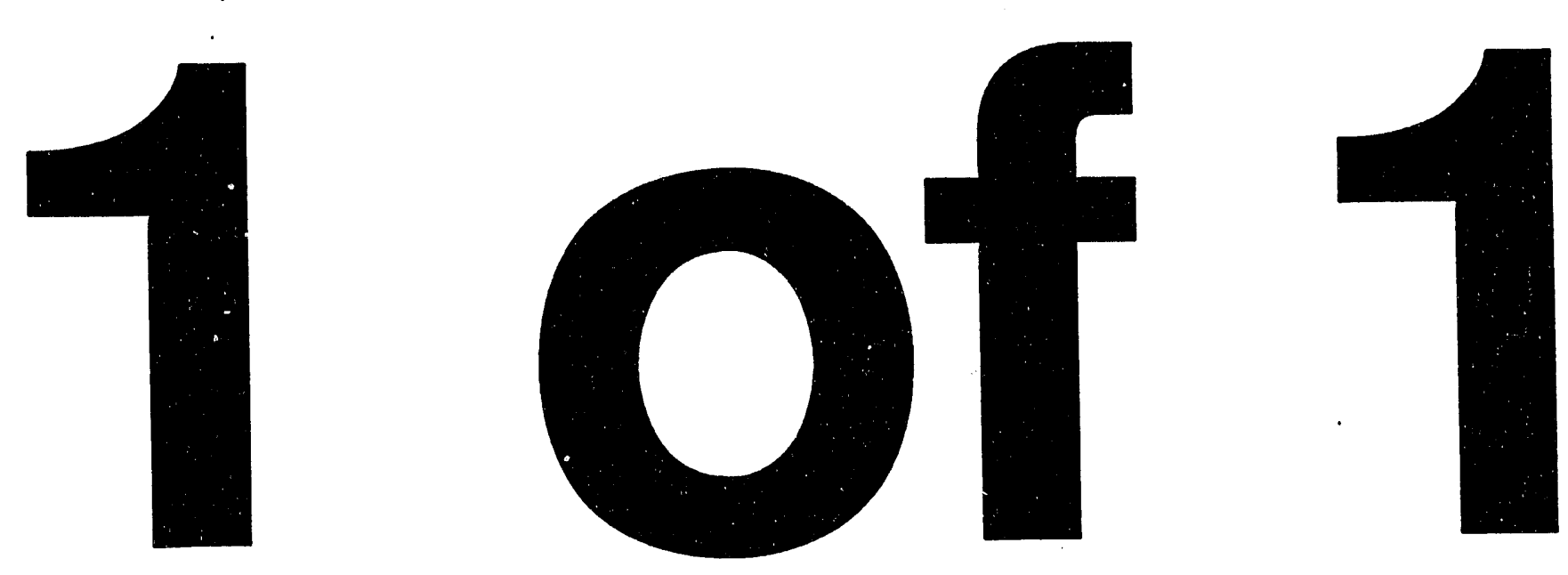


\title{
MECHANISMS FOR THE OPERATION OF THIN FILM TRANSISTORS ON FERROELECTRICS*
}

\author{
C. H. SEAGER, D. MCINTYRE, AND B. A. TUTTLE \\ Sandia National Laboratories, Albuquerque, N.M.
}

\section{J. EVANS}

Radiant Technologies Inc., Albuquerque, N.M.

\begin{abstract}
The electric field emanating from the surface of a poled ferroelectric can control the conduction properties of an overlying semiconducting film; this combination of materials can thus serve as a non-destructive readout, non-volatile memory device. Here we will describe a variety of experimental studies of these devices designed to probe the physics of their operation. The experimental systems included sputtered, n-type semiconductor (SC) films of $\mathrm{In}_{2} \mathrm{O}_{3}$ and $\mathrm{ZnO}$ deposited on bulk PLZT ferroelectrics (FE) and thin PZT FE films. Two distinctly different types of device response were measured in this study; in the first, the change in SC film conductance observed in the remanent FE state is in the direction expected from the remanent polarization vector in the ferroelectric. In the second, typically seen in thin film FE devices, the opposite behavior is observed. We find that these two general cases of behavior, including the observed variations of the SC film conductances and carrier mobilities, can be described by a general model which takes into account not only the FE displacement vector, but also charge injected from the semiconductor into the ferroelectric during biasing of the gate.
\end{abstract}

\section{INTRODUCTION}

In contrast to a Destructive-Readout (DRO) ferroelectric memory, which undergoes a change of polarization state as it is read, there exists a class of FE memories which utilize the remanent dielectric displacement to alter the conduction state of an adjacent semiconductor layer. Figure 1 illustrates a device of this type. In Figure 2 we show the electrostatic energy diagram appropriate to discussing the physics of such devices. A differential form of Gauss's law describes the relationship between 


\section{DISCLAIMER}

This report was prepared as an account of work sponsored by an agency of the United States Government. Neither the United States Government nor any agency thereof, nor any of their employees, makes any warranty, express or implied, or assumes any legal liability or responsibility for the accuracy, completeness, or usefulness of any information, apparatus, product, or process disclosed, or represents that its use would not infringe privately owned rights. Reference herein to any specific commercial product, process, or service by trade name, tradernark, manufacturer, or otherwise does not necessarily constitute or imply its endorsement, recommendation, or favoring by the United States Government or any agency thereof. The views and opinions of authors expressed herein do not necessarily state or reflect those of the United States Government or any agency thereof. 
the maximum electric field in the $\mathrm{SC}, \mathrm{E}_{\mathrm{Sc}}$, the $\mathrm{FE}$ dielectric displacement, $\mathrm{D}_{\mathrm{fe}}$, and any free charge, $Q_{i}$, which might reside at the FE/SC interface:

$$
\varepsilon_{s c} E_{s c}=Q_{i}+D_{f e}
$$

where $\varepsilon_{\mathrm{SC}}$ is the dielectric constant of the semiconductor. Depending on the sign of $D_{f e}$ and $Q_{i}$, the $S C$ layer will either be inverted, depleted, or accumulated. Figure 1 shows the case of depletion. Because $D_{f e}$ is typically a large number, values of $E_{S c}$ calculated from equation 1 are so large that it is likely that carriers will be injected into the ferroelectric. For example, if the relative dielectric constant of the $S C$ is 10 and $D_{\text {fe }}$ is $30 \mu \mathrm{C} / \mathrm{cm}^{2}, \mathrm{E}_{\mathrm{sc}}$ is $\sim 3 \times 10^{7} \mathrm{~V} / \mathrm{cm}$ ! If this injected "homocharge" becomes trapped as $\mathrm{Q}_{\mathrm{i}}, \mathrm{E}_{\mathrm{SC}}$ will drop, stopping the injection process. Thus we might anticipate that interface charge could play a role in device operation, and, in fact, other investigators studying similar devices have also emphasized the importance of this phenomena 1,2 .

A second major consideration in predicting the device response is the anticipated change in the SC film conductance as the surface potential, $V_{m}$, is altered. All the materials considered in this study are degenerately doped n-type semiconductors. Because of the high doping levels, depletion and accumulation widths are small, usually less than $100 \AA$, even for values of $\mathrm{V}_{\mathrm{m}}$ as large as several volts. This means that only a small region of the $\mathrm{SC}$ film adjacent to the ferroelectric is altered during device operation. However, as will be discussed later in this paper, carrier mobilities can be sensitive functions of grain size and doping level. This means that calculating the change in film conductance as $V_{m}$ is varied is more involved than just estimating the change in average carrier density brought about by an accumulation or depletion region near the FE/SC interface. We will show that the Hall effect can be a useful tool to understand how the average microscopic carrier mobility responds to changes in $V_{m}$ brought about by alterations in gate bias.

\section{EXPERIMENTAL}

\section{Sample preparation}

Thin film NDRO devices were fabricated at Radiant Technologies Inc.on oxidized silicon wafers. These structures consisted of a $100 \mathrm{~nm}$ layer of $\mathrm{Ti}$, a $200 \mathrm{~nm}$ Pt bottom gate electrode, a $300 \mathrm{~nm}$ PZT ferroelectric layer capped by a $\sim 40 \mathrm{~nm}$ semiconducting 
$\mathrm{In}_{2} \mathrm{O}_{3}$ film. These films were prepared by electron beam evaporation of In followed by a brief $400{ }^{\circ} \mathrm{C}$ air anneal which converted the deposited film to a semiconducting oxide. Top contacts to the SC layer were either Pt or ITO/Pt sandwiches.

NDRO test structures were also fabricated on hot-pressed $0.25 \mathrm{~mm}$ thick plates ${ }^{3}$ of bulk lead lanthanum zirconate titanate (PLZT). The semiconductor layers for these bulk FE devices were either $\mathrm{Al}$ or In doped $\mathrm{ZnO}$ or nominally undoped $\mathrm{In}_{2} \mathrm{O}_{3}$. The $\mathrm{ZnO}$ films were deposited using RF magnetron sputtering in a $1.3 \mathrm{~Pa} \mathrm{Ar}$ atmosphere. The sputtering targets were three inches in diameter pressed and sintered from $99.99 \%$ pure powders. Base pressures in the cryopumped sputtering chamber were $2-8 \times 10^{-6} \mathrm{~Pa}$ before argon introduction. The target-to-substrate distance was $5 \mathrm{~cm}$, and the substrates were rotated during deposition to promote film uniformity. The substrates were cleaned for one hour using a dual-wavelength UV-ozone cleaner and then immediately inserted into the sputtering chamber. The bottom gate and the top contacts to the SC layer were sputtered $\mathrm{Au}$ in these bulk NDRO structures. Ancillary measurements showed that these contacts introduced no appreciable resistance in measurements of the electrical properties of the SC films.

\section{Electrical measurements}

Polarization versus electric field measurements were performed with a standard Sawyer-Tower circuit in which the voltage on a series capacitor was used to monitor the displacement current of the SC/FE sandwich as the applied gate bias was ramped up and down with the usual triangular waveform. The resistance of the SC layer was measured with four terminal methods; in some cases four contacts were arranged in a square pattern at the periphery of the SC layer, and a Van derPauw analysis ${ }^{4}$ was used to calculate bulk resistivity and Hall mobility. In the case of the devices fabricated on thin film PZT, a more conventional bridge structure was used for transport determinations. The magnetic field was impressed perpendicular to the SC sample plane with peak field values of $0.77 \mathrm{~T}$, and both field directions were employed. Extensive averaging of the Hall response resulted in excellent signal to noise ratios and a minimum detectable Hall mobility of $\sim 0.001 \mathrm{~cm}^{2} / \mathrm{V} \mathrm{sec}$. During the studies of retention of the written device states, white light from a tungsten-halogen lamp was sometimes focussed on the samples; in addition, some measurements of the spectral dependence of lightinduced effects were also carried out using a zenon arc lamp/monochromator tandem. 


\section{RESULTS AND DISCUSSION}

In this section we will first focus on the general types of response seen for our thin film and bulk NDRO devices. Our survey of these properties will be largely restricted to measurements of SC film resistance versus applied gate bias. Later we will focus specifically on questions of how the SC surface band bending controls transport in the semiconductor film. In addition we shall make an attempt to quantitatively assess the amount of charge trapped at the FE/SC interface.

Device characteristics for a $\mathrm{In}_{2} \mathrm{O}_{3} /$ thin film PZT NDRO structure are shown in Figure 3. Here we have plotted the "on-state", which is the SC 4 terminal film resistance during gate bias application, and the "off state" which is the same quantity plotted after the applied gate bias has been returned to zero from the "on state" value. While the "on state" resistance decreases for positive gate biases, as you might predict from the fact that the n-type SC surface should be accumulated in this bias direction, the behavior of the hysteresis is unexpected. Based on the polarization characteristics of the ferroelectric you would expect that the SC film resistance would be higher after returning from plus gate biases, since the FE displacement vector is larger, but the reverse is seen. In addition to this anomaly in the "on state" behavior, the "off state" resistance curve is higher after writing with positive gate biases than with negative gate biases. Equation 1 and simple physical arguments show that this can only be the case if injected charge exceeds $D_{f e}$.

All of the devices that have been fabricated on thin film PZT show these "anomalous" device characteristics. However, this is type of behavior is not always seen in prototype NDRO devices made on bulk ferroelectric plates. Figure 4 shows the same device characteristics measured on a $\mathrm{ZnO} /$ bulk 7/65/35 PLZT NDRO transistor. At this composition, the FE hysteresis curves are essentially "square", as shown in Figure 5. The NDRO device characteristics are quite different from those in Figure 2; the hysteresis behavior, and the "off state" behavior are consistent with the predictions of equation 1, without the need to invoke the presence of injected charge. While the modulation of the SC resistance for this particular device is not as large as is seen in Figure 2, due probably to the particular SC resistivity and film thickness, we have found that the general shape of the resistance versus gate bias curves is reproduced for all devices made on this square-loop ferroelectric.

We can also make devices on bulk ferroelectric plates which display the "anomalous" response seen in Figure 3. This is observed when we use 9/65/35 PLZT, a pseudo-relaxor ferroelectric whose composition is near the transition region between 
ferroelectric, anti-ferroelectric, and paraelectric phases in the PLZT phase diagram 5 . Hysteresis curves obtained on capacitors fabricated from this composition show values of $P_{r}$, the remanent polarization, which are less than $2 \mu \mathrm{C} / \mathrm{cm}^{2}$. The NDRO characteristics measured on devices made with this material are shown in Figure.6. These look much like those shown in Figure 3 for the thin film PZT devices; thus, when $P_{r}$ is deliberately reduced, effects of injected charge seem to become dominant. Later we will show how the magnitude of this injected charge density may be estimated by comparing hysteresis curves obtained on metal/PZT/metal capacitors with those meaured on NDRO structures.

If our interpretation of the devices curves in Figure 3 is correct, we must address how injected charge influences device parameters like resistance throw, and retention. From our previous discussion it is clear that $\mathrm{Q}_{\mathrm{i}}$ dominates the effects of dielectric polarization in the devices made on thin FE films, since the direction of the resistance change is opposite to that expected from $D_{f e}$ alone. Accordingly, we might anticipate that retention of the written state would depend on the stability of $Q_{i}$ with time. Figure 7 shows the time evolution of the two memory states in a thin film $\left(20 / 80 \mathrm{PZT} / \mathrm{In}_{2} \mathrm{O}_{3}\right)$ NDRO device. The starting values of these curves were produced by ten second gate pulses of + or $-7 \mathrm{~V}$, and the on (-gate bias) and off (+ gate bias) resistance values of the semiconductor were monitored while the device was in the dark. There is a slow decay of both states that roughly fits a $\log (t)$ dependence. A series of measurements (not shown) indicates that this decay process is only a very weak function of temperature. It is however strongly accelerated in the presence of small gate biases, or white light, as shown in Figures 8 and 9 . After only $\sim 10$ seconds, the two memory states have actually crossed each other in the illuminated device.

Why does this reversal occur? We suggest that photocarriers produced either in the SC or the FE layer cancel most or all of the trapped interface charge, leaving only the effect of the ferroelectric polarization. In effect this restores the device to "normal" operation where the electric field from the Fe displacement vector dominates. We have also obtained capacitance data (not shown) on these same NDRO structures that show that the SC depletion layer width in the "on" and "off" states undergoes a similar reversal with time under illumination. This data was obtained at $500 \mathrm{~Hz}$, a frequency low enough that the SC sheet resistance did not distort the measurement of the SC depletion layer/FE capacitor sandwich.

The general behavior shown in our retention studies on thin film devices suggests that the decay of the "on" or "off" device states is consistent with the tunneling of charge into or out of traps near the FE/SC interface. The $\log (t)$ time dependence 6 the 
lack of any strong variation with temperature, and the sensitivity of the rate to small biases all are consistent with this explanation. The last effect is expected because tunneling is exponentially sensitive to the width of the forbidden barrier, and for the case where the SC is depleted, this barrier certainly changes with gate bias. In the accumulated case, the fact that injected charge is stable for long times seems to indicate that the states which trap $Q_{j}$ are at least $40-80 \AA$ deep in the ferroelectric.

Several types of measurements have been carried out to understand the nature of the conduction process in the $\mathrm{In}_{2} \mathrm{O}_{3}$ and $\mathrm{ZnO}$ films used in these devices. Although a more detailed set of experiments is now underway to improve our understanding, our preliminary measurements are sufficiently informative to help us understand some of our observed device behavior. It should first be pointed out that each type of SC film has distinctly different responses to post growth anneals, and these are indicative of the differences in the chemical stability of the donor centers. In the case of $\operatorname{In}_{2} \mathrm{O}_{3}$, the asdeposited film shows a very weak variation of resistivity with temperature consistent with that of metallic In; the measured Hall mobility at least partially follows this variation showing that the carrier concentration changes only weakly with temperature. Data from films deposited on glass are shown in Figure 10. After a $400^{\circ} \mathrm{C}$ air anneal the In is converted to $\mathrm{In}_{2} \mathrm{O}_{3}$ causing the films to become much more resistive, but the mobility rises significantly. This is shown in Figure 11 . A further $200^{\circ} \mathrm{C}$, hotplate treatment for 5 minutes reverses the drop in carrier concentration and raises the Hall mobility. These effects are summarized in Table 1, below.

\section{Table $1300 \mathrm{~K}$ Transport Coefficients on $\operatorname{In}_{2} \mathrm{O}_{3}$ films}

\section{Resistivity}

$(\Omega \bullet c m)$

As-received

$400^{\circ} \mathrm{C}$ oven anneal

$200^{\circ} \mathrm{C}$ on hotplate
0.011

0.33

0.0165
Mobility

(cm²/Vesec)

$0.93 \pm 0.1$

$5.8 \pm 0.6$

$30.6 \pm 0.5$
Carrier Density

$\left(\mathrm{cm}^{-3}\right)$

$1.28 \times 10^{21}$

$6.28 \times 10^{18}$

$2.39 \times 10^{19}$

These measurements show how sensitive these films are to oxygen ambients, and that the behavior of the transport properties is complex. In interpreting all of our Hall mobilities we are relying on past transport studies, which show that, in general the carrier concentrations in polycrystalline films can be deduced from the formula 7 :

$$
n=r /\left(\mu_{H} e \rho\right)
$$


where $\mu_{\mathrm{H}}$ is the Hall mobility, $\rho$ is the resistivity, $\mathrm{e}$ is the electronic charge, and $\mathrm{r}$ is a factor which is 1.93 for scattering from ionized impurities. While $\mathrm{n}$ deduced from equation 2. is usually a good approximation to the true electron density in the conduction band, $\mu_{\mathrm{H}}$ is only an effective mobility ${ }^{8}$. This is because grain boundaries in these films offer a substantial resistance to carrier flow, and the measured mobility is a complicated response function of carrier scattering in the bulk and at grain boundaries.

Given this caveat, what does the data in Figures 10 and 11 and in Table 1, tell us about transport in these films? The fact that $n$ changes so much with annealing seems to indicate that the presence of oxygen controls the effective donor density. The increase in mobility after the $400^{\circ} \mathrm{C}$ anneal is consistent with the conversion of metallic In to $\mathrm{In}_{2} \mathrm{O}_{3}$. One might initailly think that ionized donor scattering would control $\mu_{\mathrm{H}}$ in the converted films, but the rise in mobility coupled with the rise in $\mathrm{n}$ after the brief $200^{\circ} \mathrm{C}$ treatment is totally at odds with this explanation. However, this result is consistent with many past studies which indicate that higher dopant densities reduce the width of potential barriers at grain boundaries and can significantly raise the probability that carriers will tunnel across these boundaries 9 .

The $\mathrm{Al}$ and In doped $\mathrm{ZnO}$ films show similar carrier concentrations and Hall mobilities, but their response to thermal anneals in air is quite different from that of $\mathrm{In}_{2} \mathrm{O}_{3}$. An example of the temperature dependence of resistivity and Hall mobility for an $\mathrm{Al}$ doped $\mathrm{ZnO}$ film is shown in Figure 12. As in the $\operatorname{In}_{2} \mathrm{O}_{3}$ case, $\rho$ is weakly decreasing function of temperature, and most of this decrease can be attributed to the variation of carrier mobility. To check for the kind of behavior seen in the $\operatorname{In}_{2} \mathrm{O}_{3}$ samples, some of these films were also annealed in air at various temperatures. A summary of some of these results for $\rho, n$, and $\mu_{H}$ is given in Table 2, below:

Table 2 Transport Coefficients for Al doped $\mathrm{ZnO}$

$\begin{array}{lccc}\begin{array}{l}\text { Film } \\ \text { (Temp. of Meas.) }\end{array} & \begin{array}{c}\text { Resistivity } \\ (\Omega \bullet \mathrm{cm})\end{array} & \begin{array}{c}\text { Mobility } \\ \left(\mathrm{cm}^{2} / \mathrm{V} \bullet \mathrm{sec}\right)\end{array} & \begin{array}{c}\text { Carrier Density } \\ \left(\mathrm{cm}^{-3}\right)\end{array} \\ \text { As-deposited } & 0.027 & 1.58 \pm 0.02 & 2.83 \times 10^{20} \\ \ldots . .(\text { at 295K) } & & & \\ . . " \text {..( at 40K) } & 0.0365 & 1.24 \pm 0.02 & 2.67 \times 10^{20} \\ 300^{\circ} \text { Annealed } & 0.97 & .048 \pm 0.004 & 2.59 \times 10^{20} \\ \ldots . . .(\text { at 295K) } & & & \end{array}$

Here, as in Table 1, we have assumed in our calculation of $n$ that, $r$, the ratio of the Hall mobility to the conductivity (drift) mobility is 1.93 . This number is appropriate for 
carriers which primarily scatter off of ionized impurities ${ }^{7}$ (donors), but may not be strictly correct for scattering at grain boundaries. Notice that the response to an air anneal is quite different to that seen for $\mathrm{In}_{2} \mathrm{O}_{3}$. In this case the carrier density is virtually unchanged by the anneal, while the mobility drops noticeably. It is possible that this effect may be caused by thermally induced segregation of a small fraction of the aluminum dopant to the grain boundaries followed by the formation of a highly resistive $\mathrm{Al}_{2} \mathrm{O}_{3}$ intergranular barrier layer. Alternatively, oxygen chemisorbed at these boundaries may change the effective trap state density. In any case, unlike $\operatorname{In}_{2} \mathrm{O}_{3}$, the population of active donors seems insensitive to these air anneals.

Preliminary results on $\mathrm{In}_{2} \mathrm{O}_{3}$ films show that film resistivities and mobilities are rather independent of thickness. However, for the $\mathrm{ZnO}$ films, our data indicates that both the conductivity and carrier mobility are functions of film thickness, increasing strongly as the film thicknesses are varied in the 400-1000 $\AA$ region. As an example, the sheet resistance of $600 \AA$ films of In or Al doped $\mathrm{ZnO}$ deposited on bulk PLZT is typically $1 / 3$ of that found for $400 \AA$ films. These trends are seen on films deposited on both glass and ferroelectric substrates. This effect has been reported by other workers ${ }^{10}$ for thicker films of $\mathrm{ZnO}$, and appears to be indicative of an evolution of the film microstructure. It is not uncommon to find that the average grain size in polycrystalline semiconductor films increases with distance from the nucleation interface as more energetically unfavorable grain habits are crowded out, and the film microstructure becomes simpler with fewer defects per unit volume. If electronic transport is limited by narrow potential barriers at the grain boundaries, the average resistivity will be higher near the nucleation interface, translating to an observable thickness dependence of the transport properties. This particular feature will have important consequences for $\mathrm{ZnO}$ based NDRO device operation, since the field emanating from the FE substrate only penetrates some $50-150 \AA$ into the semiconductor. In effect this type of device (at these doping densities) is controlling transport in a region where the local resistivity is already large, and the other regions of the SC film are acting as an undesirable shunt resistor.

Hall mobility and 4 terminal resistivity were also measured on the semiconducting films on actual NDRO devices constructed on both thin film PZT and bulk PLZT. In the former case residual leakage through the PZT prevents mobility determinations during the application of gate bias, but the transport properties could still be accurately measured in the remanent state. These measurements were carried out on several types of bulk FE NDRO devices, many of which had only small (a few percent) resistance differences (device throw) between the + and - gate bias remanent states. For these 
small throw devices, it was sometimes observed that both $n$ and $\mu_{H}$ varied with gate bias polarity; these variations, coupled with the fact that the accuracy of the Hall mobility was usually $+/-0.5 \%$, made it difficult to draw firm conclusions about the influence of gate bias on SC film transport. However, all of the thin film PZT devices, and a few of the samples made on bulk PLZT exhibited resistance throws of at least a factor of two. Transport measurements on these high-throw devices always yielded the same results, and it is this data that we shall concentrate on in this paper. Figure 13 shows carrier concentration and mobility data of this type for an NDRO device on 7/65/35 PLZT. This device displays "normal" NDRO behavior where the resistance appears to dominated by the ferroelectric displacement vector. In this experiment the gate bias was cycled sequentially between the values displayed on the abscissa. A surpising result is that the mobility, not the carrier concentration, is the parameter influenced by the gate bias. Several thin film PZT NDRO devices, which display the "anomalous" response indicative of charged surface states, were also characterized with Hall measurements. Thes data also showed that to first order, resistance changes could be attributed almost totally to changes in carrier mobility.

If one presumes that the SC film has properties which are uniform both in the device plane and normal to it, these transport results seem odd, because the logical supposition is that gate bias alterations either add (in accumulation) or subtract (in depletion) a layer of carriers near the FE/SC interface. One would then expect that the mobility would stay constant, and the carrier density would be the parameter observed to change with gate bias. However, we already suspect from the temperature, thickness, and annealing dependence of resistivity and mobility, that these SC films are not uniform electrically, and, in particular, it is probable that high resistance regions exist at the grain boundaries. Under these circumstances, we expect that the primary effect of altering the local carrier density might be to alter the grain boundary resistance, and hence the effective carrier mobility. For instance, in a degenerate semiconductor, the width of a tunneling barrier at a grain boundary is very sensitive to the electron density. So we might expect that with gate biases which induce depletion, a small region of the SC film will have no carriers at all, forcing current up to the top of the film (which is probably very conductive anyway). On the other hand, with gate biases causing accumulation, the flood of carriers pulled to the surface will drastically narrow the grain boundary tunneling barriers there, causing a large redistribution of current in the SC film. The key point is that, if most of the resistance to carrier flow is at the grain boundaries, gate bias alterations will always alter the observed carrier mobility, since that parameter is a measure of the grain boundary effects. Thus, these data appear to 
require the hypothesis that grain boundary scattering dominates transport. Unfortunately, a detailed prediction of the actual size of these effects requires much more knowledge of film microstrucure and grain boundary properties than is accessible from our electrical measurements.

Finally we discuss the results of experiments designed to partially test the selfconsistency of the interface state model; as a byproduct of these, we can also make estimates of the amount of charge trapped near the FE/SC interface during gate bias cycling. When a bias is applied to the gate of a FE/SC NDRO sandwich structure some of this bias is dropped across the semiconductor. If the hysteresis curves are measured on such structures and compared to those observed on metal/FE/metal capacitors, distinct differences in response should be observed. In principle it is possible to use this type of data to estimate the SC band bending and interface charge density in the NDRO devices. Figure 14 shows data of this type taken on equal area $\mathrm{Pt} / \mathrm{PZT} / \mathrm{Pt}$ and $\mathrm{In}_{2} \mathrm{O}_{3} / \mathrm{PZT} / \mathrm{Pt}$ capacitors fabricated on the same wafer. In this particular case, displacement currents were measured for identical gate voltage cycles, and the displacement current response of the NDRO capacitor is clearly smaller. For purposes of the following analysis it would be desirable to have data on the Pt/PZT/Pt structure which is taken with a gate bias cycle which produces the same displacement current excursion as observed on the NDRO structure. In lieu of this information, we have scaled the Pt capacitor data proportionately to simulate this information. While this is not a strictly correct procedure, it probably allows us to obtain sufficiently accurate estimates of the desired parameters; this scaled curve is shown as the dashed line in Figure 14. By measuring the voltage offset between the NDRO data and the scaled Pt curve we can estimate, at any value of displacement vector, the voltage dropped across the semiconducting layer. Operationally this is effected by drawing a horizontal line across the data plot and noting the voltage difference between the two curves. The results are shown in Figure 15; in carrying out this procedure, we are assuming that any displacement current due to charge tunneling into the $\mathrm{FE} / \mathrm{SC}$ interface is negligible compared to $\mathrm{D}_{\mathrm{fe}}$. The curves shown in figure 15 seem to agree with the $\mathrm{SC}$ resistance plots for a similar NDRO device shown in Figure 3. At large positive gate biases the SC resistance is at a minimum corresponding to accumulation of the SC surface. However, after the gate bias is returned to zero, the device resistance has increased drastically, approaching values $\sim 2 / 3$ as large as the - gate bias, high resistance state. Likewise, $V_{m}$ has crossed through zero approaching a value $\sim 1 / 2$ of the fully depleted condition. Similarly, negative gate bias exposure followed by a return to zero bias leaves $V_{m}$ at a positive value. Thes curves are clearly consistent with gate bias application injecting 
like-sign charge (homocharge) from the SC into the interface. This charge is largely retained at zero-bias, and it produces values of the SC surface potential opposite to those present during application of the bias. An almost complete reversal of the gate bias is required to replace this interface charge with charge of the opposite sign.

If the SC doping level is known, it is possible to calculate $Q_{i}$ at any applied gate bias by using equation 1 , and the $V_{m}$ values in Figure 15. This is done by introducing the relationships between $E_{s c}$ and $V_{m}$ which are well known for accumulation and depletion regions in degenerately doped semiconductors. We have implemented this procedure numerically for several chosen doping levels appropriate to our $\mathrm{In}_{2} \mathrm{O}_{3}$ films. No attempt was made to account for any charge located at defects in the semiconductor (such as grain boundaries). Figure 16 shows the values of $\mathrm{Q}_{\mathrm{i}}$ computed using a donor density of $10^{19} \mathrm{~cm}^{-3}$ and the known value of the conduction band density of states measured in the limit of weak doping. The conduction band state density affects the results because it determines the spatial distribution of the accumulation layer for positive surface potentials. This curve (solid circles) gives sensible values of $Q_{i}$ in depletion, but the result that the charge density gets more negative as the surface potential is decreased from $-1.5 \mathrm{~V}$ is contrary to one's intuition. If anything, $Q_{i}$ should stay constant or become slightly more positive as $\mathrm{V}_{\mathrm{m}}$ becomes more negative. However, one important feature of the SC electronic structure has been omitted from this calculation. At doping levels above $2-3 \times 10^{18} \mathrm{~cm}^{-3}$ it is known that the donor levels are strongly interacting; they form a donor band which merges with the conduction band ${ }^{11}$. Under these circumstances, the bottom of the conduction band becomes smeared out and the effective state density drops drastically. To simulate this effect, which is quite difficult to calculate theoretically, we have recalculated $Q_{i}$ using density of states values 5 (solid triangles) and 25 (solid squares) times smaller than the unperturbed conduction band value. Both of these revised curves show an evolution of $\mathrm{Q}_{i}$ which is more in line with our expectations. The magnitude of the trapped interface charge is large, about $6-9 \times 10^{13} \mathrm{~cm}^{-3}$, but this is of course required if $Q_{i}$ is to cancel the effect of $D_{f e}$ in Equation 1. Because of the approximations involved in these calculations, we regard these deconvolution results as support for the general concepts in the model, but hardly ironclad proof of specific details. It is fairly clear, however, that the hysteresis data in Figure 14 give strong evidence for voltage drops in the semiconductor, and the sign of these drops at various stages of the gate bias cycle are inconsistent with $V_{m}$ being controlled by $D_{f e}$ alone. These data plus the sign and hysteresis direction of the device characteristics in Figure 3. clearly demonstrate the importance of charge injection in these thin film NDRO memories. 


\section{CONCLUSIONS}

We have demonstrated that two distinctly different types of response can be obtained from NDRO memory devices. The "normal" response, where the effects of FE polarization alone control the semiconductor film resistance, is seen in devices fabricated on square loop bulk ferroelectrics. The "anomalous" response, characterized by injection and trapping of large amounts of charge from the $\mathrm{SC}$, is seen in thin film PZT devices or bulk FE devices where $P_{r}$ is small. Further work is required to elucidate whether the dominance of injected charge in thin film devices is primarily due to the presence of more traps in the ferroelectric, or to the smaller ratio of $\mathrm{P}_{\mathrm{r}}$ to the full field polarization in these thin films. Our side-by-side measurements of metal/FE/metal capacitors and NDRO capacitors have demonstrated that the there are clear indications of voltage drops across SC depletion and accumulation regions in biased NDRO structures. The SC surface potential versus gate bias plots obtained from this data have been deconvoluted to obtain estimates of the charges trapped in the FE during NDRO biasing. These charge densities can reach values of $10-15 \mu \mathrm{C} / \mathrm{cm}^{2}$; the fact that they remain in the $\mathrm{FE}$ for extended periods when the $\mathrm{SC}$ is accumulated seems to require that the filled traps are at least 40-80 $\AA$ from the SC/FE interface.

Our measurements of the charge transport properties of the semiconductor component of these devices have shown that all these degenerately doped n-type materials appear to have carrier mobilities which are limited by grain boundary scattering effects. Because this scattering process is very sensitive to the local carrier concentration, the modulation of SC film resistance in the biased NDRO structures appears as a mobility change in our Hall effect measurements. Other features of the conduction properties of these films which impact NDRO behavior are the thickness dependence of film resistivity, and in the case of the $\mathrm{In}_{2} \mathrm{O}_{3}$, a dependence of resistivity on air anneals indicative of a oxygen-defect origin for the donor states.

\section{*ACKNOWLEDGEMENTS}

This work was supported under DOE contract\#DE-AC04-94AL85000. Note that, because of time limitations, much of the information on the properties of the $\operatorname{In}_{2} \mathrm{O}_{3}$ and $\mathrm{ZnO}$ films was not presented orally at the ISIF conference. 


\section{REFERENCES}

1. S. Y. Wu, IEEE Trans. Electron Devices 21, 499 (1974).

2.N. Maffei and S. B. Krupanidhi, J. Appl. Phys. 74, 7551 (1993).

3 Motorola Inc.,Ceramics Products Division, Albuquerque, N.M..

4 L. J. Van derPauw, Phillips Res. Reports 13, 1 (1958).

5 G. H. Haertling and C. E. Land, J. Amer. Ceram. Soc.54, 1-11 (1971).

$6 \mathrm{~S}$. Manzini and A. Modelli, Insulating Films on Semiconductors( ed. by J. F. Verwey and D. R. Wolters, Elsevier, 1983), p. 112.

7. S. M. Sze Physics of Semiconductor Devices (Wiley-Interscience, 1969), p. 45.

8. H. P. Maruska, A. K. Ghosh, A. Rose, and T. Feng, Appl. Phys. Lett. 36, 381 (1980).

9. G. E. Pike and C. H. Seager, J. Appl. Phys. 52, 3960 (1979).

10. Y. Qu, T. A. Gessert, K Ramanathan, R. G. Dhere, R. Noufi, and T. J. Coutts, J. Vav. Sci. Technol. A11, 996 (1993).

11.See for instance, B. I Halperin and M. Lax, Phys. Rev. 148, 772 (1966). 


\section{FIGURE CAPTIONS}

FIGURE 1. A schematic of a semiconductor/ferrcelectric NDRO device.

FIGURE 2. The electrostatic energy diagram for electrons in a degenerately doped semiconductor deposited on a ferroelectric. Electron affinity differences between the two materials have been ignored in this picture.

FIGURE 3. The variation of 4 terminal semiconductor resistance with gate bias for a thin film PZT NDRO device measured at room temperature. The filled circles are values measured with the gate bias on. The filled diamonds represent data taken after exposure to the gate bias shown on the abscissa but with the gate shorted to one of the semiconductor top electrodes.

FIGURE 4. The 4 terminal semiconductor resistance versus gate bias for an Al doped $\mathrm{ZnO}$ film/ bulk 7/65/35 PLZT NDRO device at $233 \mathrm{~K}$. The "on state" (filled circles) is measured with the gate bias on. The "off state" (filled diamonds) is obtained after relaxing the gate bias to zero.

FIGURE 5. The dielectric displacement charge measured versus gate bias for a In doped $\mathrm{ZnO}$ film/bulk 7/65/35 PLZT NDRO device. The device area was $\sim 09 \mathrm{~cm}^{2}$.

FIGURE 6. The variation of 4 terminal semiconductor resistance with gate bias for an Al doped $\mathrm{ZnO}$ film/bulk 9/65/35 PLZT NDRO device measured at $294 \mathrm{~K}$. The "on state" (filled circles) is measured with the gate bias on. The "off state" (filled diamonds) is obtained after relaxing the gate bias to zero.

FIGURE 7. The relaxation of the 4 terminal resistance for an $\mathrm{In}_{2} \mathrm{O}_{3} /$ thin film PZT NDRO device measured at $300 \mathrm{~K}$ with no illumination and at zero gate bias. The high resistance state was created with a 5 second $+7 \mathrm{~V}$ gate pulse which ended at time zero on the plot, and the low resistance data were taken after a 5 second $-7 \mathrm{~V}$ gate pulse. The data displayed have been offset by subtracting 5000 ohms.

FIGURE 8. The effect of small gate biases on the relaxation of the high resistance (pulsed with + gate bias) state of a $\operatorname{In}_{2} \mathrm{O}_{3} /$ thin film PZT NDRO device measured at $300 \mathrm{~K}$ with no illumination. The gate bias was relaxed to zero for the actual measurement. Similar data (not plotted here) show that these same biases also have important effects on the relaxation of the low resistance (pulsed with - gate bias) state.

FIGURE 9. The effect of white light illumination on relaxation of the 4 terminal resistance for a $\operatorname{In}_{2} \mathrm{O}_{3} /$ thin film PZT NDRO device. Light appears to cause the device 
to relax to a state where the ferroelectric polarization is controlling the semiconductor resistance.

FIGURE 10. The temperature variation of the 4 terminal resistivity of an as-deposited $\mathrm{In}_{2} \mathrm{O}_{3}$ film. Hall mobilities obtained at several temperatures are also shown. Film thickness was $\sim 800 \AA$.

FIGURE 11. The temperature variation of the 4 terminal resistivity of an $\operatorname{In}_{2} \mathrm{O}_{3}$ film annealed in air at $400^{\circ} \mathrm{C}$. Hall mobilities obtained at several temperatures are also shown. Film thickness was $\sim 800 \AA$.

FIGURE 12. The temperature variation of the 4 terminal resistivity of an as-deposited Al doped $\mathrm{ZnO}$ film. Hall mobilities obtained at several temperatures are also shown. Film thickness was $\sim 2000 \AA$.

FIGURE 13. Hall mobilities and carrier concentrations measured for an In doped $\mathrm{ZnO}$ semiconductorfilm deposited on a bulk 7/65/35 PLZT plate. The sample was electroded to form a NDRO device, and the transport coefficients were measured at different gate biases; these are plotted in temporal sequence on the abscissa. The SC film thickness was $\sim 400 \AA$, and the donor dopant was In.

FIGURE 14. Hysteresis data for $\mathrm{Pt} / \mathrm{PZT} / \mathrm{Pt}$ and $\mathrm{In}_{2} \mathrm{O}_{3} / \mathrm{PZT} / \mathrm{Pt}$ capacitors fabricated on the same Si wafer. The dashed curve is a congruently scaled version of the Pt/PZT/Pt data used to estimate the SC bias drop and interface charge densities.

FIGURE 15. The $\operatorname{In}_{2} \mathrm{O}_{3}$ surface potential during gate biasing estimated from the hysteresis data shown in Figure 13.

FIGURE 16. The interface charge injected during biasing of an $\mathrm{In}_{2} \mathrm{O}_{3} /$ thin film PZT NDRO device estimated from the data of Figure 14. Filled circles show calculated $Q_{i}$ values for the unperturbed density of conduction band states. Filled triangles and squares are for conduction band densities 5 and 25 times smaller; these later values account for the merging of the conduction and donor bands at these high doping densities. 


\section{SEMICONDUCTOR/ FERROELECTRIC}

NON-DESTRUCTIVE READOUT MEMORY DEVICE

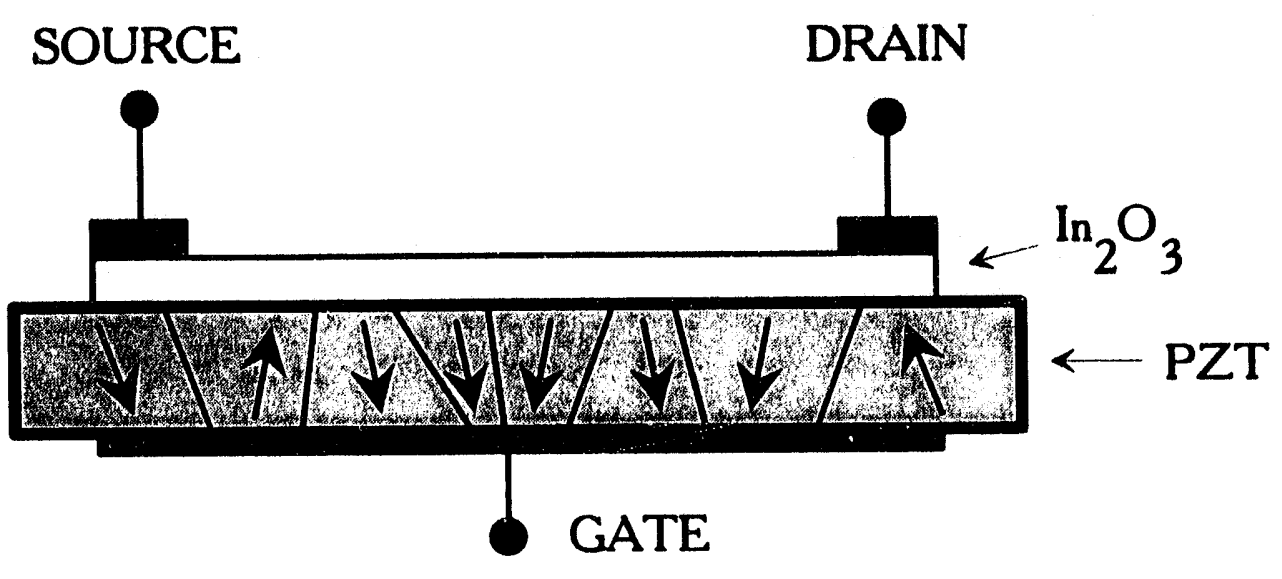




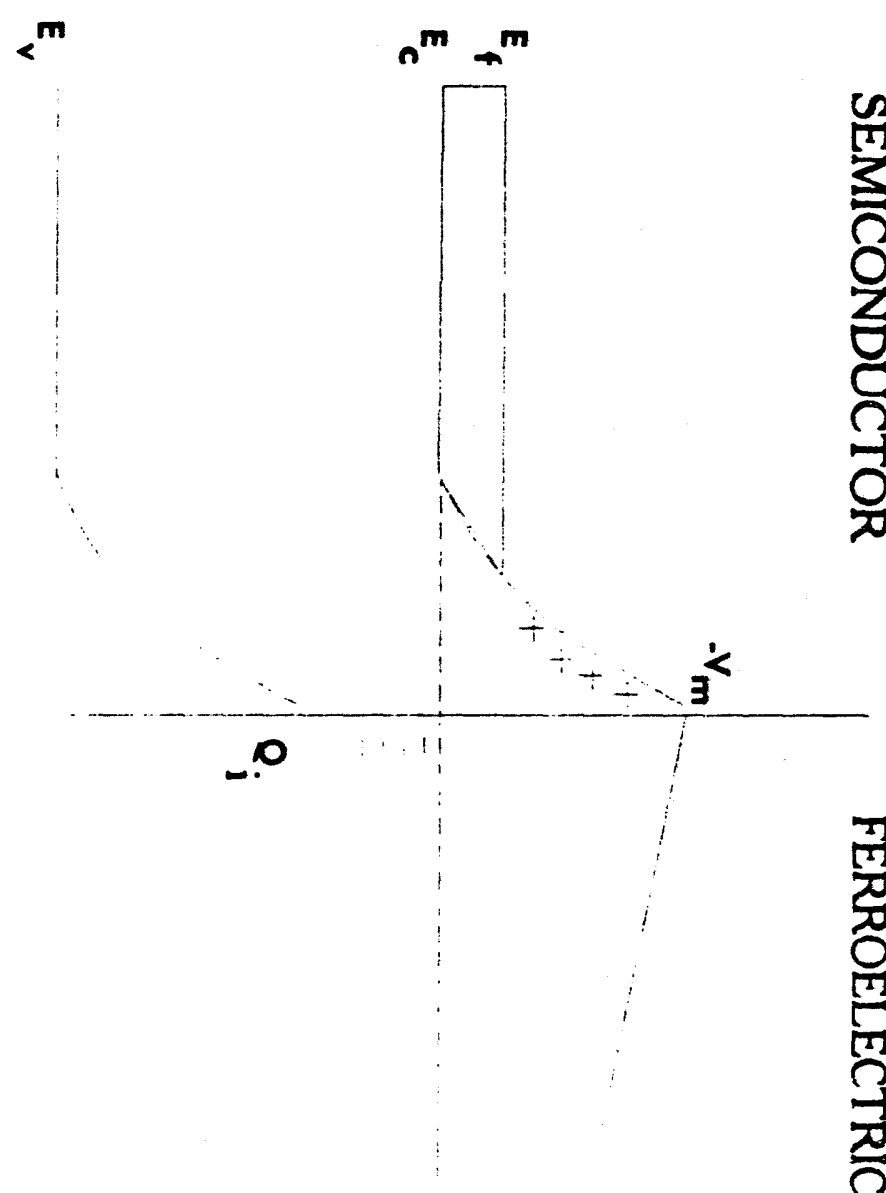

ㅇ

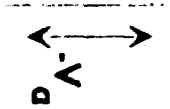




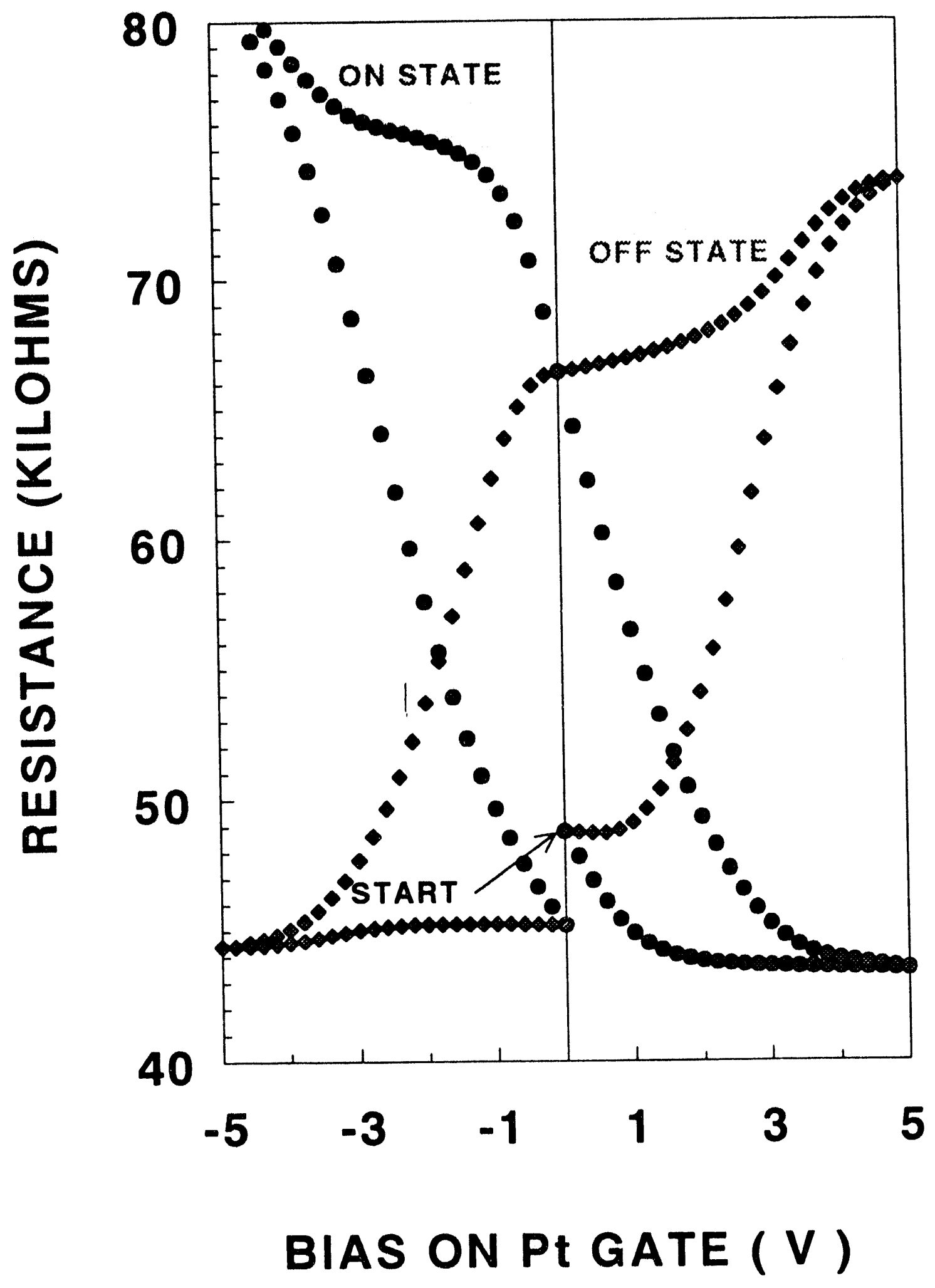




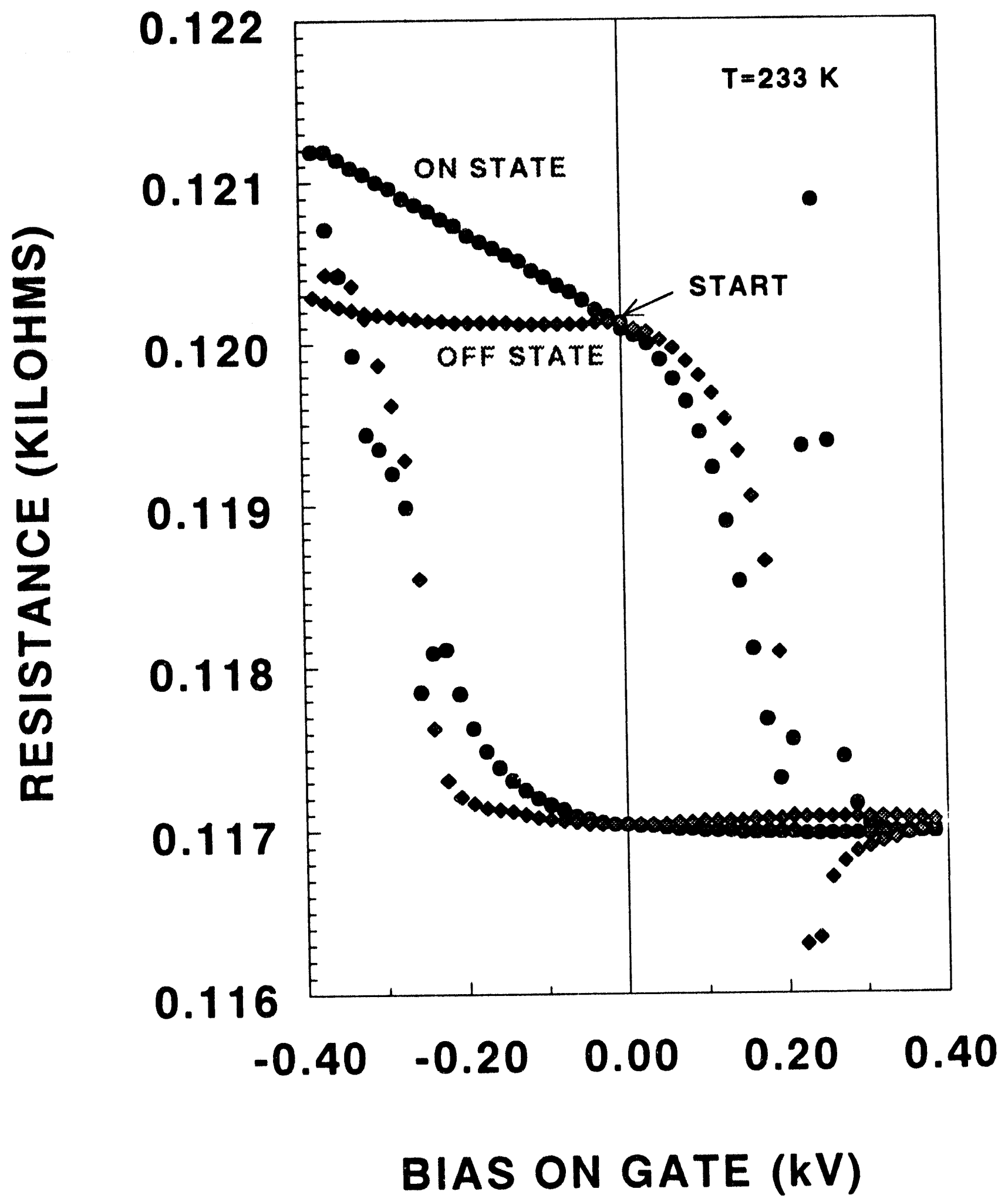


CHARGE $(\mu \mathrm{C})$

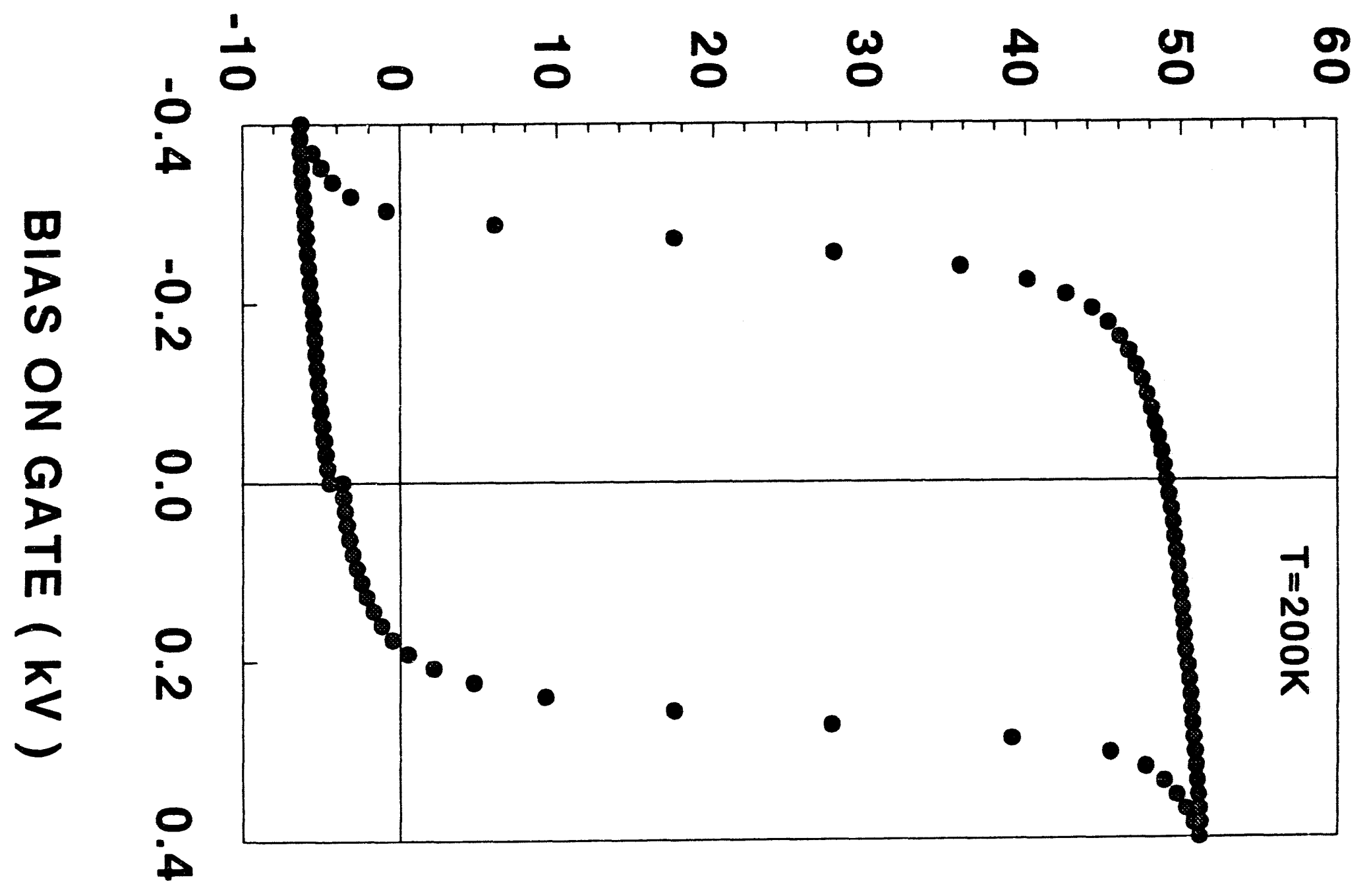




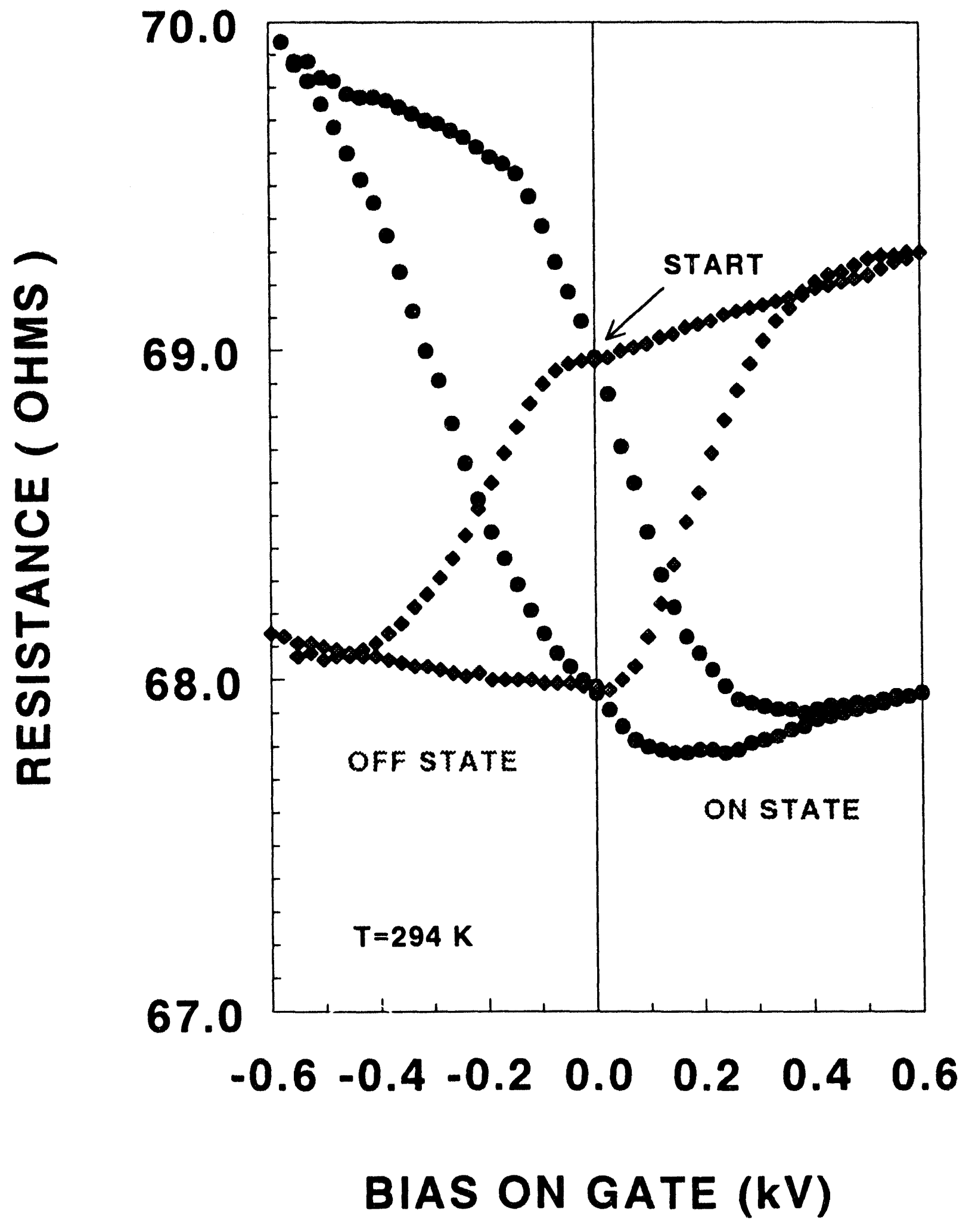




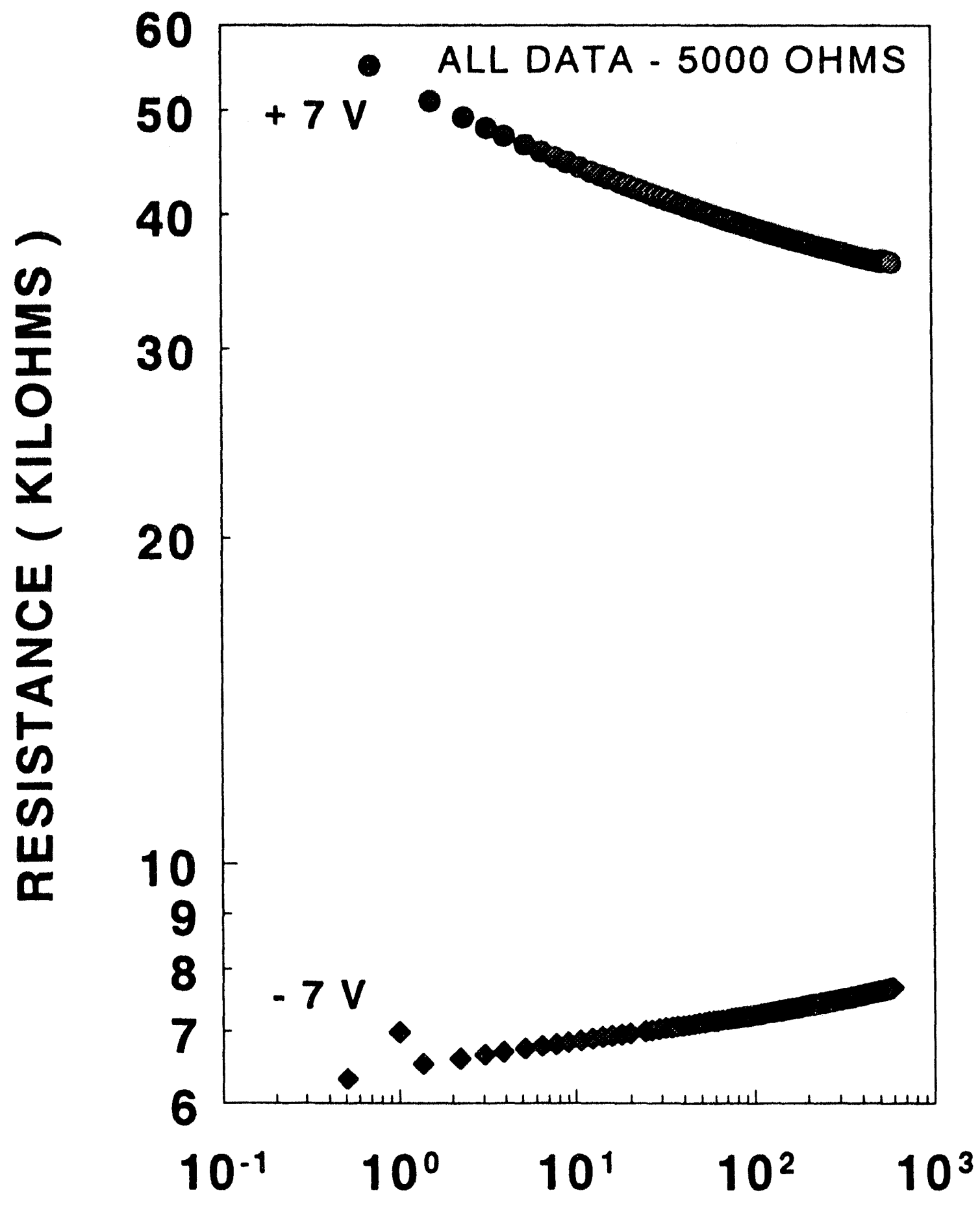

TIME (SEC) 


\section{RESISTANCE ( KILOHMS )}

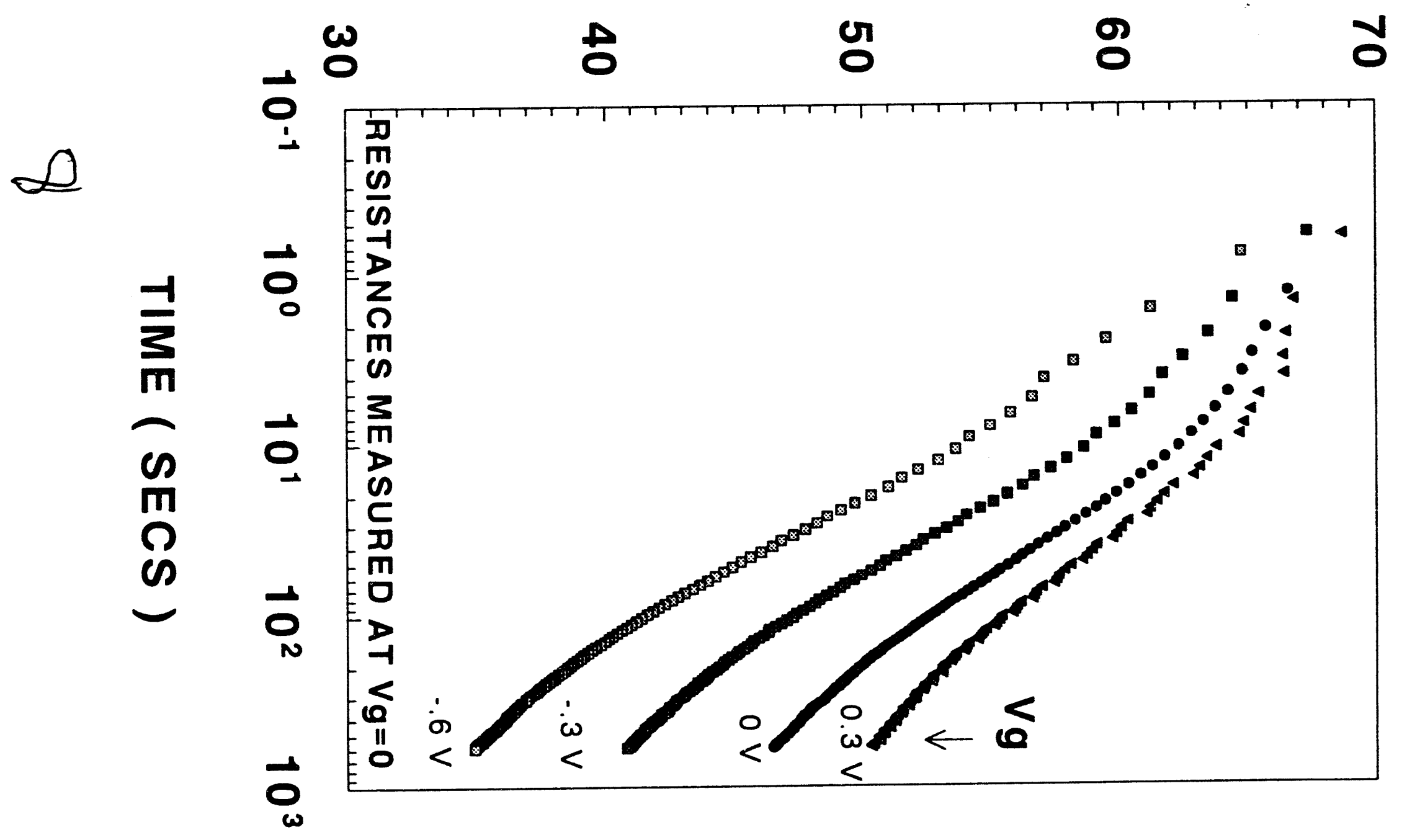




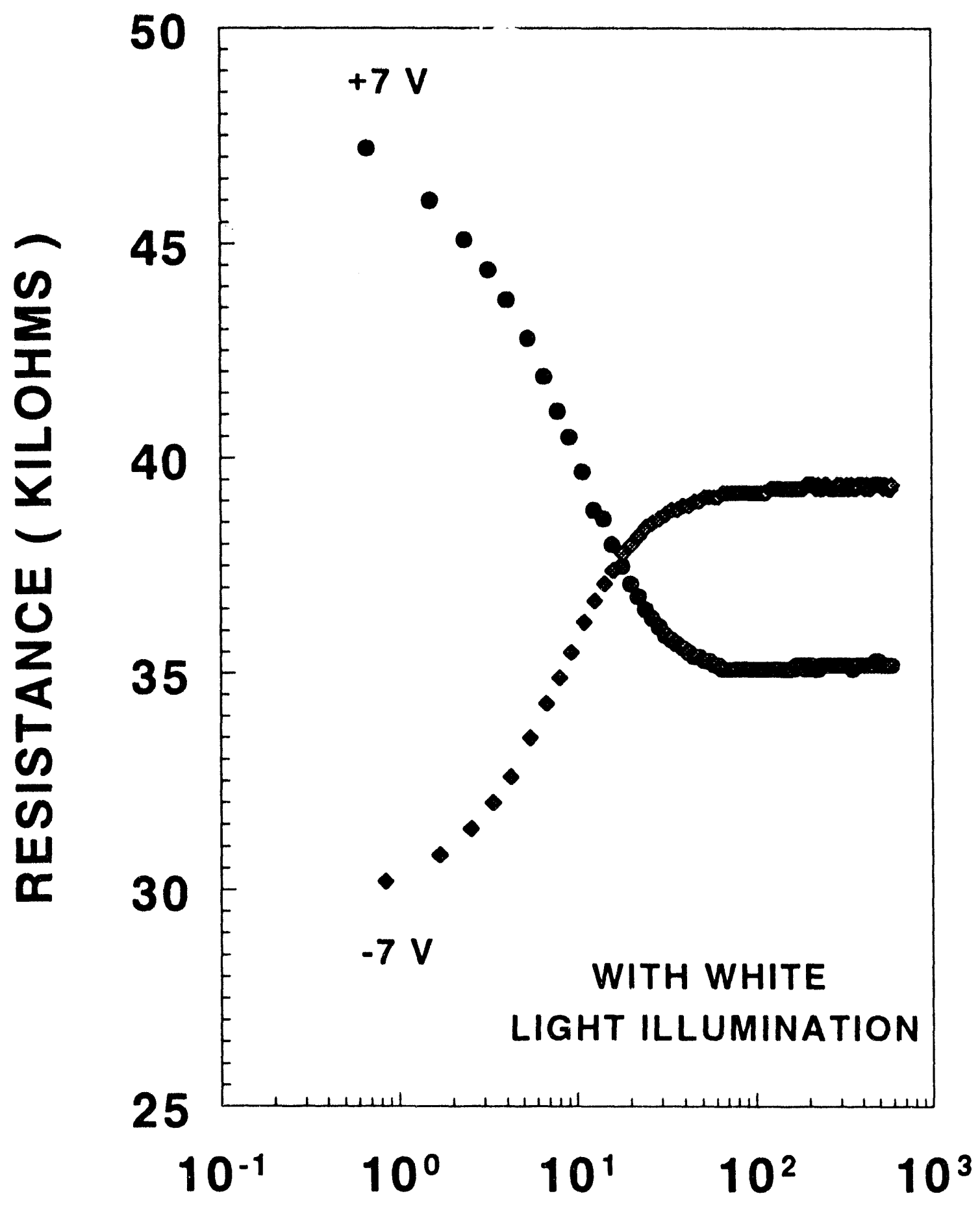

TIME (SECS ) 


\section{RESISTIVITY (OHM.CM)}

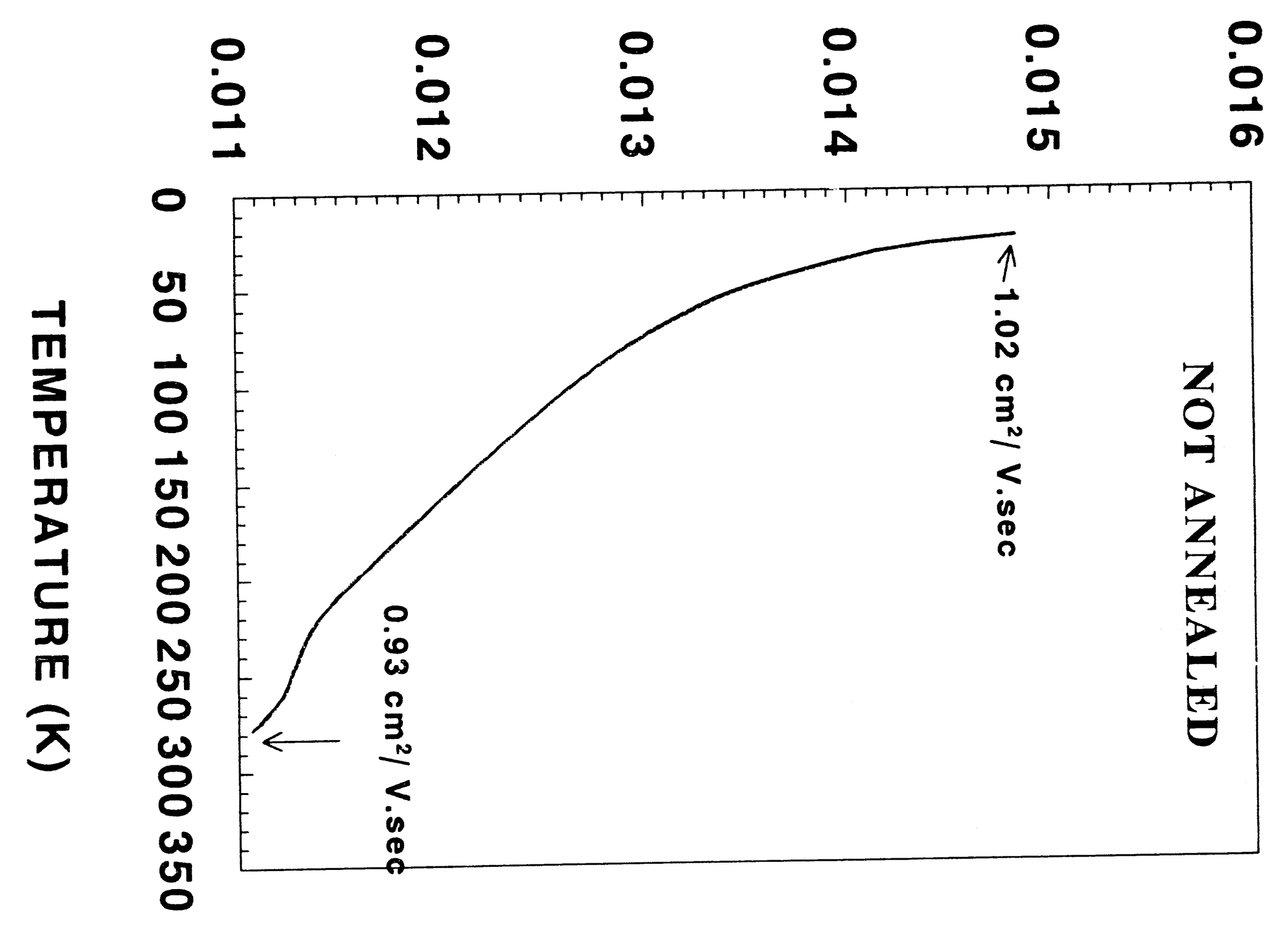


RESISTIVITY (OHM.CM)

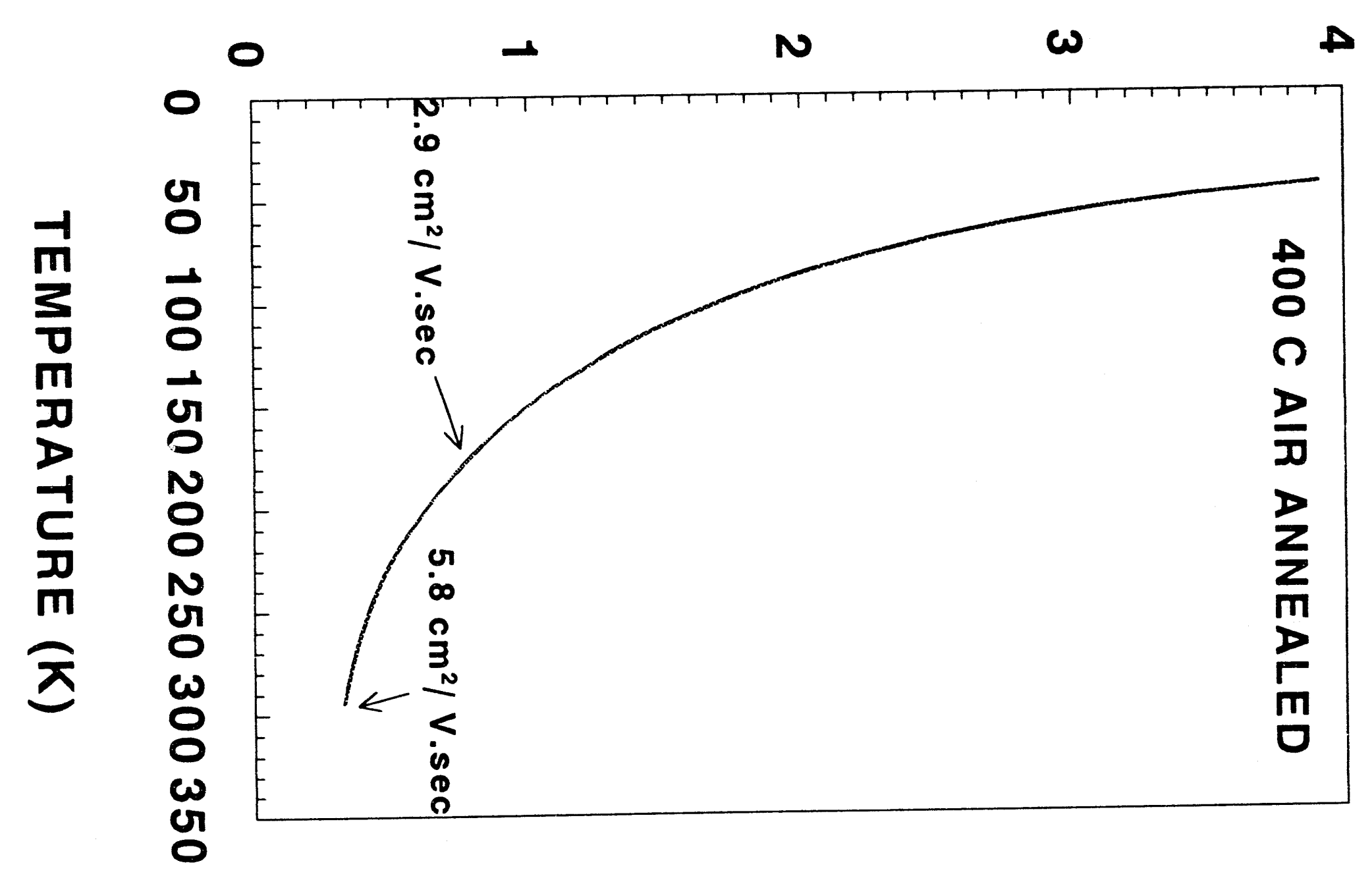




\section{RESISTIVITY (OHM.CM)}

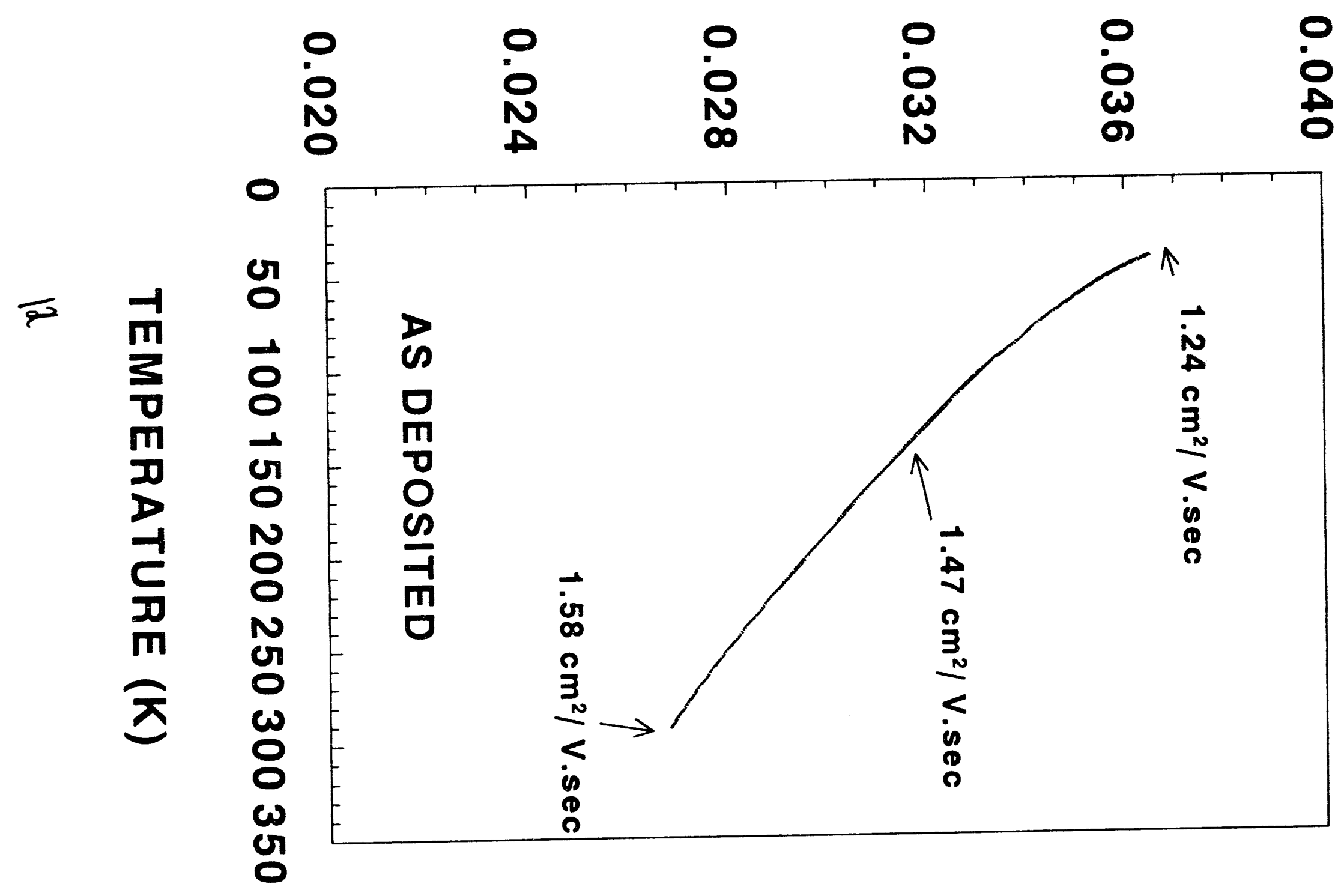




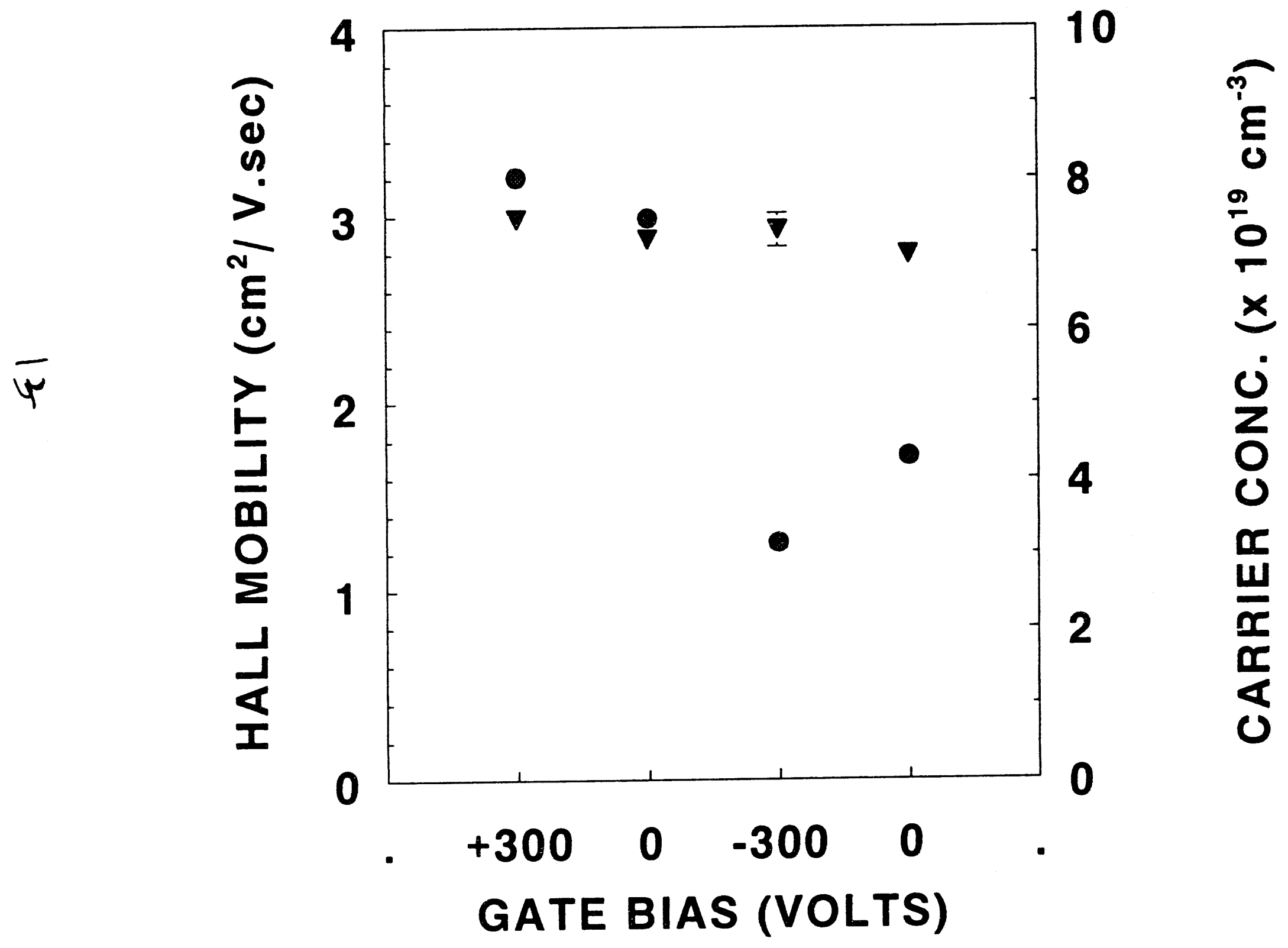




\section{$($ słon ) s}

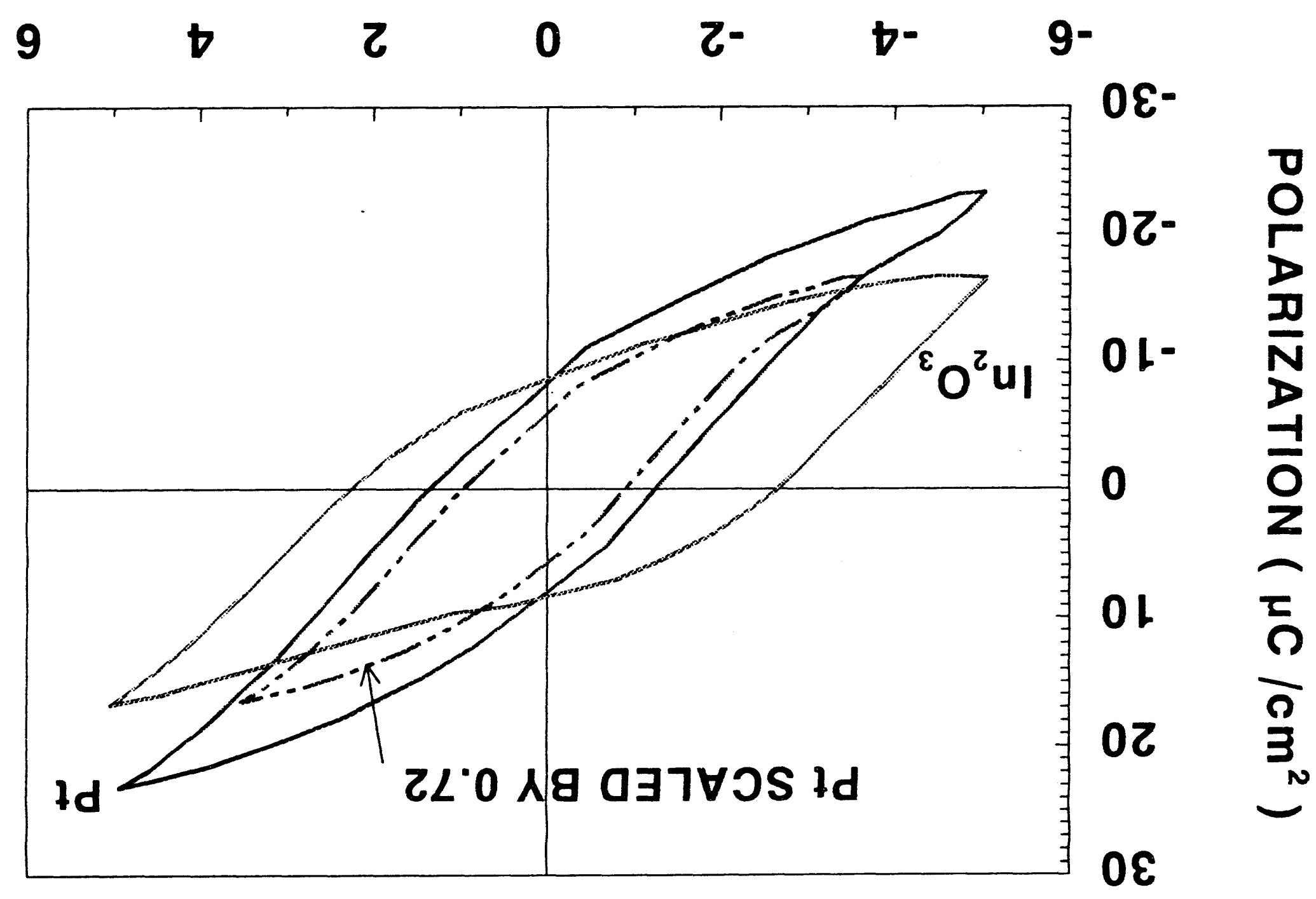




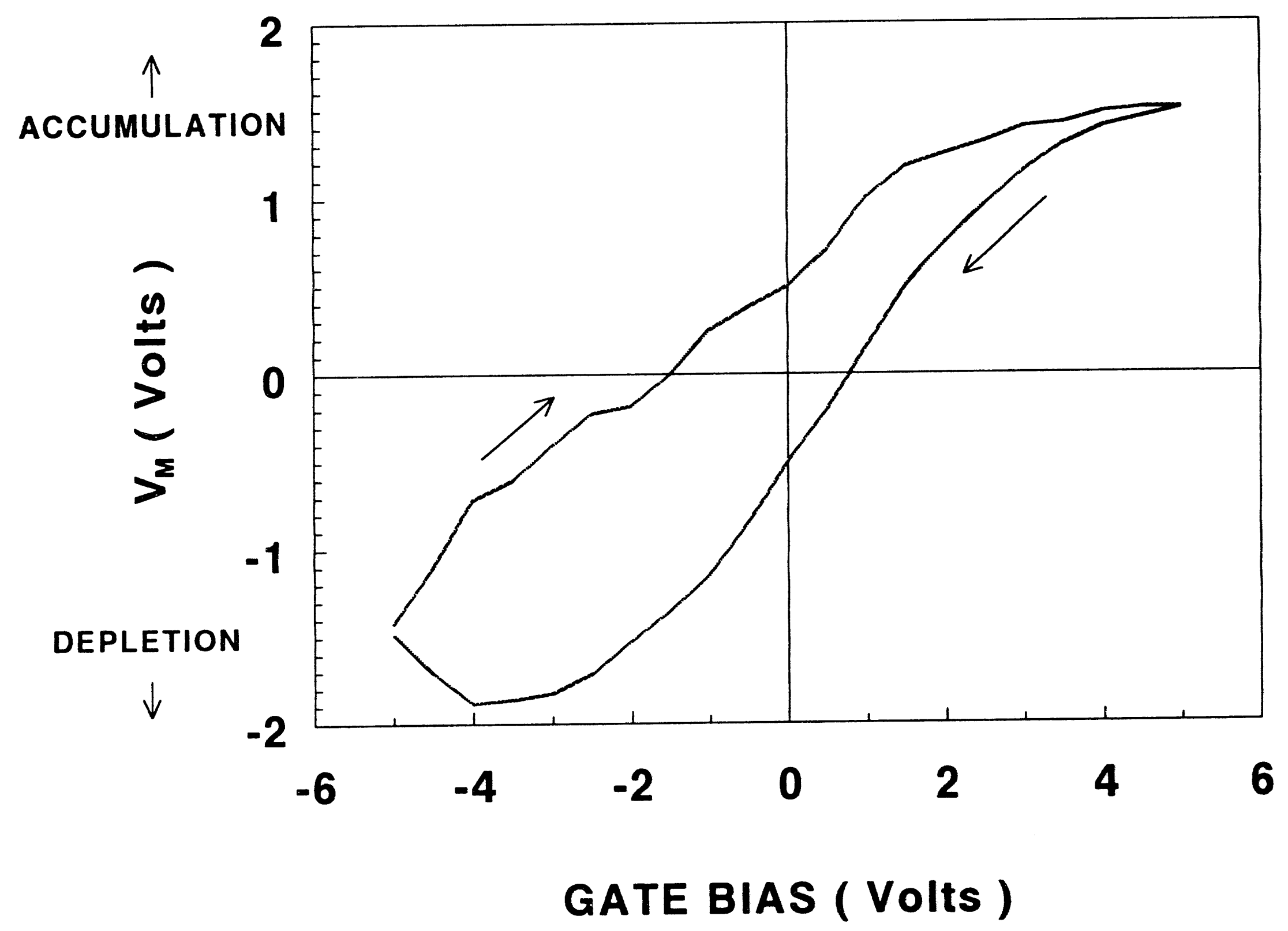




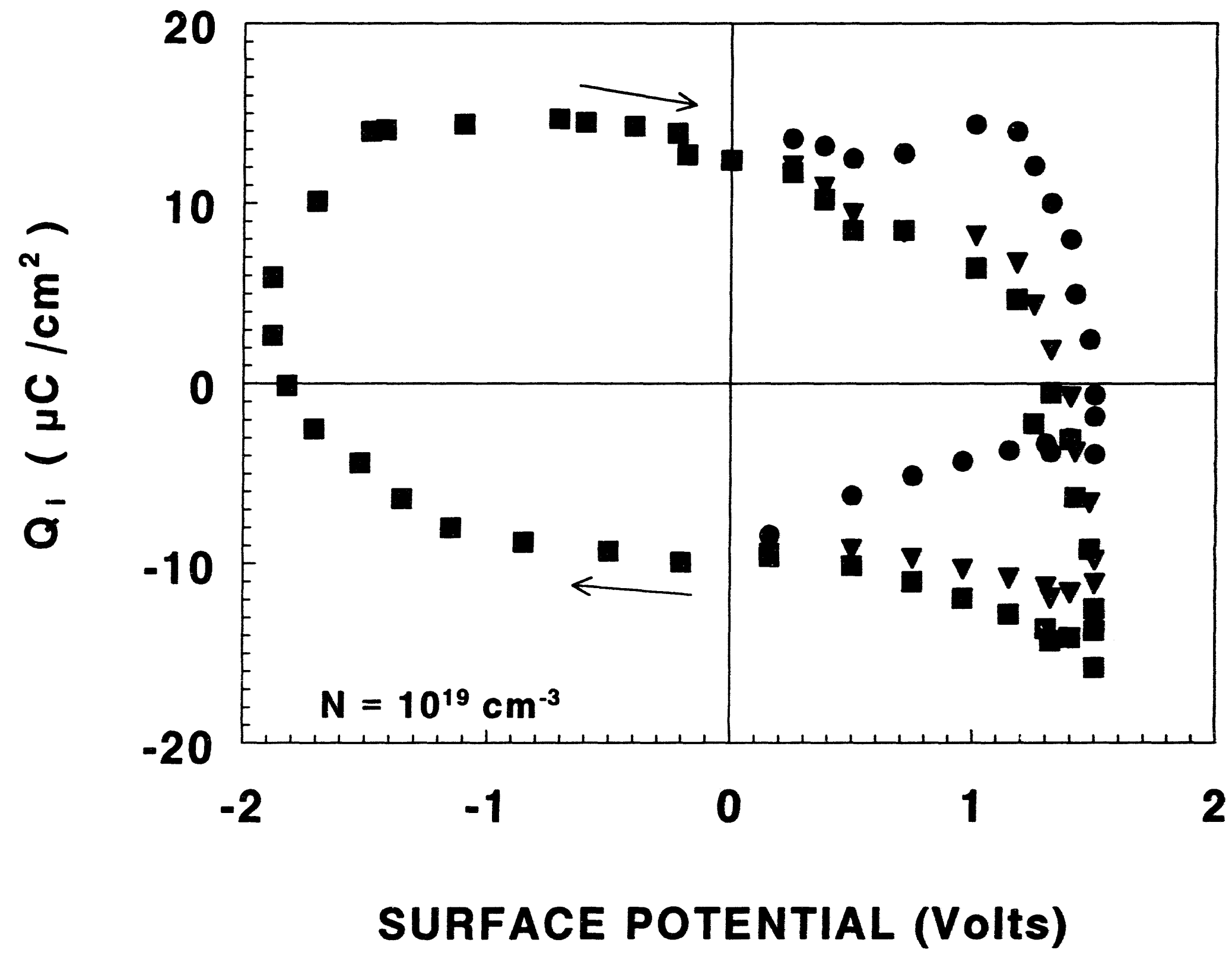



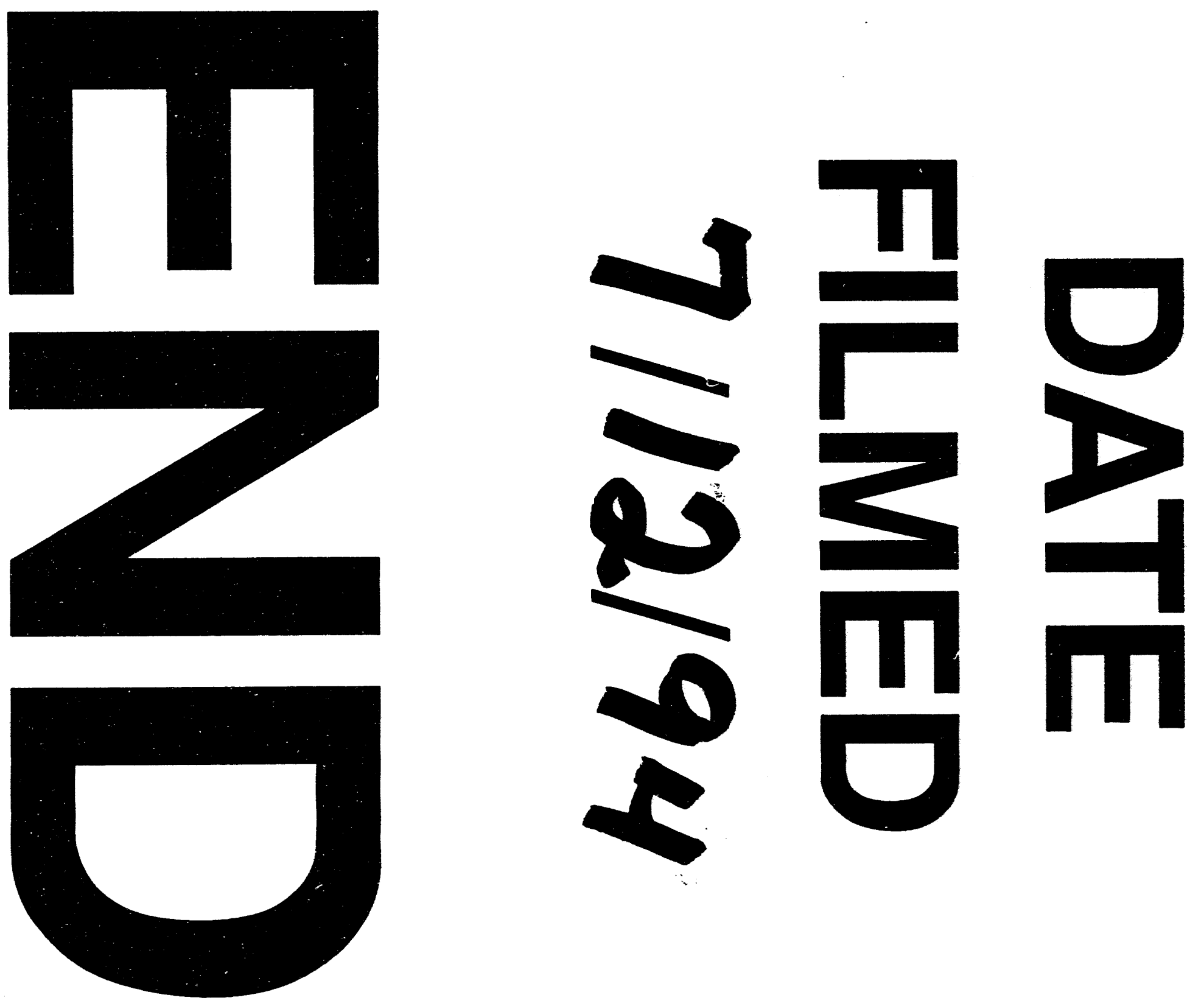

5 
